\title{
Extended dust shell of the carbon star U Hydrae observed with AKARI
}

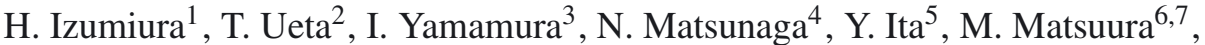

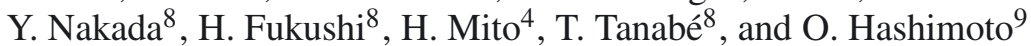

1 Okayama Astrophysical Observatory (OAO), National Astronomical Observatory of Japan (NAOJ) ${ }^{\star}, 3037-5$ Honjo, Kamogata, Asakuchi, Okayama 719-0232, Japan e-mail: izumiura@oao.nao.ac.jp

2 Department of Physics and Astronomy, University of Denver, 2112 E. Wesley Ave., Denver, CO 80208, USA e-mail: tueta@du.edu

3 Institute of Space and Astronautical Science, Japan Aerospace Exploration Agency, 3-1-1 Yoshinodai, Chuo-ku, Sagamihara, Kanagawa 252-5210, Japan e-mail: yamamura@ir.isas.jaxa.jp

${ }^{4}$ Kiso Observatory, Institute of Astronomy, Graduate School of Science, The University of Tokyo, 10762-30 Mitake, Kiso, Nagano 397-0101, Japan e-mail: [matsunaga;mito]@kiso.ioa.s.u-tokyo.ac.jp

5 Astronomical Institute, Graduate School of Science, Tohoku University, 6-3 Aramaki Aoba, Aoba-ku, Sendai, Miyagi 980-8578, Japan e-mail: yita@astr.tohoku.ac.jp

${ }^{6}$ UCL-Institute of Origins, Astrophysics Group, Department of Physics and Astronomy, University of College London, Gower Street, London WC1E 6BT, UK

e-mail: mikako@star.ucl.ac.uk

7 UCL-Institute of Origins, Mullard Space Science Laboratory, University of College London, Holmbury St. Mary, Dorking, Surrey RH5 6NT, UK

8 Institute of Astronomy, Graduate School of Science, The University of Tokyo, 2-21-1 Osawa, Mitaka, Tokyo 181-0015, Japan e-mail: nakada@kiso.ioa.s.u-tokyo.ac.jp; [hina@;ttanabe]@ioa.s.u-tokyo.ac.jp

9 Gunma Astronomical Observatory, 6860-86 Nakayama, Takayama, Agatsuma, Gunma 377-0702, Japan e-mail: osamu@astron.pref.gunma.jp

Received 28 May 2010 / Accepted 12 November 2010

\begin{abstract}
Context. Low- to intermediate-mass stars lose a significant fraction of their mass while they are on the asymptotic giant branch (AGB). This mass loss is considered to determine the final stages of their evolution. The material ejected from the stellar photosphere forms a circumstellar envelope in its surroundings. Layers of circumstellar envelope constitute the footprint of mass-loss history.

Aims. Our aim is to probe the mass-loss history in the carbon star U Hya in the last $\sim 10^{4}$ years by investigating the distribution of dust in the circumstellar envelope with high spatial resolution.

Methods. We observed U Hya in the far-infrared (FIR) at 65, 90, 140, and $160 \mu \mathrm{m}$ simultaneously, using the slow scan observing mode of the far-infrared surveyor (FIS) aboard the infrared astronomical satellite AKARI. It produced a map of $\sim 10^{\prime} \times 40^{\prime}$ in size in each band.

Results. The FIS maps reveal remarkably circular, ring-like emission structure almost centered on the star, showing the presence of a detached, spherical dust shell. A hollow dust shell model gives the inner radius $R_{\text {in }}$ of $101-107^{\prime \prime}\left[(2.5-2.6) \times 10^{17} \mathrm{~cm}\right]$, thickness that covers a half of the total dust mass $\Delta R_{\mathrm{hm}}$ of 16-23" [(3.8-5.6) $\left.\times 10^{16} \mathrm{~cm}\right]$, which gives $\Delta R_{\mathrm{hm}} / R_{\mathrm{in}} \sim 0.2$, and the power-law index of the dust opacity distribution of $1.10-1.15$. The dust mass in the shell is well-constrained to be $(0.9-1.4) \times 10^{-4}\left(\kappa_{100} / 25\right)^{-1} M_{\odot}$, where $\kappa_{100}$ is the dust absorptivity at $100 \mu \mathrm{m}$ in units of $\mathrm{cm}^{2} \mathrm{~g}^{-1}$. The dust mass-loss rate at $R_{\text {in }}$ is found to be $(1.8-$ 9.6) $\times 10^{-8}\left(\kappa_{100} / 25\right)^{-1}\left(v_{\mathrm{e}} / 15\right) M_{\odot} \mathrm{yr}^{-1}$, which shows that the total mass-loss rate in the shell is at least an order of magnitude higher than the current rate, where $v_{\mathrm{e}}$ is the outflow velocity at $R_{\text {in }}$ in units of $\mathrm{km} \mathrm{s}^{-1}$. An extension of FIR emission along PA $\sim-70^{\circ}$ is found out to $\sim 5^{\prime}$ from the star, which is probably a ram-stripping wake by the ISM wind. We also find excess FIR emission that might indicate the earliest departure from spherical symmetry in the AGB mass loss inside the shell.

Conclusions. The dust shell of U Hya is a hollow sphere and has an effective width that is narrower than the spatial resolution of the FIS. It could be formed as either a direct consequence of a thermal pulse, a result of two-wind interaction induced by a thermal pulse, a termination shock, or any combination of these processes.
\end{abstract}

Key words. stars: AGB and post-AGB - stars: carbon - circumstellar matter - stars: mass-loss - stars: evolution stars: individual: U Hya 


\section{Introduction}

Low- to intermediate-mass stars with initial masses of $\sim 0.8-8 M_{\odot}$ suffer heavy mass loss during their evolution on the asymptotic giant branch (AGB) to become white dwarfs. The mass-loss rates are typically $\sim 10^{-8}$ to $10^{-4} M_{\odot} \mathrm{yr}^{-1}$ based on radio and infrared observations (Knapp \& Morris 1985; Bedijn 1987). Because the mass-loss rate exceeds the interior nuclear burning rate during the AGB evolution, the mass loss determines the fate of each AGB star by lowering the highest reachable luminosity and lifetime of the $\mathrm{AGB}$, which reduces the mass range of supernova progenitors.

Details and the mechanism(s) of AGB mass loss still remain uncertain, while it is considered to be the result of an interplay among the low surface gravity, pulsation, and radiation pressure on dust in the extended atmosphere. Empirical formulae are widely used to describe the AGB mass loss in numerical calculations (see Willson 2000, and references therein), while a new type of hydrodynamical simulations has been developed to reproduce the mass loss from AGB stars without an ad hoc massloss prescription (Gail \& Sedlmayr 1987; Bowen 1988; Fleischer et al. 1992; Höfner \& Dorfi 1997; Woitke 2006).

There is ample observational evidence that the AGB mass loss varies significantly with time on several different time scales as exemplified in IRC+10216 (Groenewegen 1997; Groenewegen et al. 1998; Mauron \& Huggins 2000). Relating the mass-loss variation to the variations in other stellar parameters would provide clues to the mechanism of the AGB mass loss, which may further be associated with the interior physical processes. For example, a thermal pulse can modulate mass loss significantly (e.g., Vassiliadis \& Wood 1993) and do so either directly or indirectly because it affects the surface luminosity, as well as the surface chemical composition, which would influence the atmospheric structure and dust formation.

Long term variation in the AGB mass loss may be traced in the structure of extended circumstellar envelopes of gas and dust. In particualr, far-infrared (FIR) observations from space permit us to investigate cold dust in the region farther than $0.1 \mathrm{pc}$ away from the central star, while such a region cannot be traced well by molecular gas because of the photodissociation by the interstellar radiation field. Early IRAS studies of evolved stars have revealed several extended dust shells in the FIR in spite of the large IRAS detector apertures of $1.5^{\prime} \times 5^{\prime}$ and $3^{\prime} \times 5^{\prime}$ at $60 \mu \mathrm{m}$ and $100 \mu \mathrm{m}$, respectively (Hacking et al. 1985; Rowan-Robinson et al. 1986). The studies further suggest there is a hollow structure in some of the extended dust shells. Such hollow, extended dust shells are consistent with the excess FIR emission in those stars.

Extended hollow dust shells may be the interaction region where the mass-loss wind is running into the interstellar medium (Rowan-Robinson et al. 1986; Young et al. 1993b; Zijlstra \& Weinberger 2002; Libert et al. 2007). Van der Veen \& Habing (1988) have, however, suggested a discontinuity in the mass-loss process that should produce a hollow dust shell to account for the observed excess FIR emission in AGB stars, noting a possible relation to a thermal pulse. Willems \& de Jong (1988) successfully modeled the distribution of AGB stars on the IRAS 12-25-60 $\mu \mathrm{m}$ two-color diagram and the presence of stars with large excess emission at $60 \mu \mathrm{m}$. They introduced a scenario that an interruption of the preceding high mass-loss phase by a thermal pulse had caused the detached dust shells in optically bright carbon stars, which are in less active mass-loss states currently. They

\footnotetext{
* A member of the National Institutes of Natural Sciences.
}

deduced that the mass-loss rate had been higher by an order of magnitude in the previous high mass-loss phase than in the post low mass-loss period.

Further investigations were made to reveal the structure of extended circumstellar shells that should reflect the mass-loss behavior in those stars. In particular, detached gas shells were discovered in the stars with excess FIR emission through the mm-wave CO lines (Olofsson et al. 1988, 1990). They concluded the occurrence of a short period of a high mass-loss phenomenon for the production of the detached gas shells. Some extended dust shells were also resolved to show actually detached structure in the FIR (Waters et al. 1994; Izumiura et al. 1996, 1997), which also suggested a sudden decrease in mass loss by more than an order of magnitude on a short time scale in the last 10000 years.

These observations stimulated both theoretical and observational studies. Numerical hydrodynamical simulations in the past decade or so shed light on the evolution of circumstellar matter produced by the mass loss from thermally pulsing AGB (TPAGB) stars (Steffen et al. 1998; Steffen \& Schönberner 2000; Mattsson et al. 2007) and its interaction with the interstellar matter (Villaver et al. 2002; Wareing 2007). New-generation infrared satellites equipped with state-of-the-art instruments, the Spitzer Space Telescope (Werner et al. 2004) and the infrared astronomical mission AKARI (Murakami et al. 2007), have brought further progress in the mapping observations of cold extended dust shells: a bow shock nebula as a result of the interaction between the stellar wind and the interstellar matter revealed in an M-type Mira variable R Hya (Ueta et al. 2006), an astropause identified in the FIR in the Mira ( $o$ Cet) system (Ueta 2008), another bow shock nebula discovered in an Mtype Mira variable R Cas (Ueta et al. 2010), and the possible wake of stellar wind in the interstellar matter wind (Geise et al. 2010). Most recently, superb spatial resolutions of the Herschel Space Observatory (Pilbratt et al. 2010) have revealed two additional detached dust shells in the FIR (Kerschbaum et al. 2010). However, high-resolution, high dynamic-range maps of the extended circumstellar envelopes of AGB stars are still scarce in the FIR.

In this paper we present the results of our far-infrared observations of an AGB star with the Far-Infrared Surveyor (FIS, Kawada et al. 2007) onboard AKARI. The star, U Hya $(\mathrm{RA}=10: 37: 33.2725, \mathrm{Dec}=-13: 23: 04.354, \mathrm{~J} 2000)$ is one of the brightest carbon stars in the optical $(V \sim 5.4, \Delta V \sim 0.5)$ with the variable type, period, and spectral type as SRb, 450 days, and C6.5, 3(N2)(Tc), respectively, in the General Catalogue of Variable Stars (Samus et al. 2007). It hosts a moderate mass loss of $1.2 \times 10^{-7} M_{\odot} \mathrm{yr}^{-1}$ with a gas outflow velocity of $6.9 \mathrm{~km} \mathrm{~s}^{-1}$ derived from mm-wave $\mathrm{CO}$ line observations (Olofsson et al. 1993). In this star technetium (Tc) absorption line has been detected (Peery 1971). Because ${ }^{99} \mathrm{Tc}$ alone can be produced by the s-process among the technetium isotopes and its half-life is $2.1 \times 10^{5} \mathrm{yr}$, the star is thought to be a bona fide object that experienced the third dredge-up presumably caused by a thermal pulse in the He-buring shell within a few $10^{5}$ years. Other s-process elements are also enhanced in this star (Utsumi 1985; Abia et al. 2002). U Hya is, thus, a true AGB star.

$\mathrm{U}$ Hya has entries at 60 and $100 \mu \mathrm{m}$ in the IRAS Small Scale Structure Catalog (Joint IRAS Science Working Group 1988 ) in spite of its current moderate mass loss and the large IRAS detector apertures. In high-resolution IRAS images based on a maximum entropy image-reconstruction technique the farinfrared emission has a ring-like structure at $60 \mu \mathrm{m}$ with a radius of $\sim 120^{\prime \prime}\left(3 \times 10^{17} \mathrm{~cm}\right)$ (Waters et al. 1994). In the followings 
we present our FIS/AKARI observations of U Hya in the farinfrared. We describe the observation procedure in Sect. 2, results and a simple model analysis in Sect. 3, and discuss the analysis results in Sect. 4.

\section{Observations}

We observed U Hya with the Far-Infrared Surveyor (FIS, Kawada et al. 2007) in the pointed observation mode of AKARI (Murakami et al. 2007) as part of an AKARI mission program, "Excavating Mass-Loss History in the Extended Dust Shells of Evolved Stars (hereafter "MLHES", P.I.: I. Yamamura)", on 2007 June 07 (UT) (OBSID = "1711504_001"). This observing program, MLHES, is a far-IR imaging survey of the circumstellar envelopes of evolved stars. An overview of the survey program will be presented in a forthcoming paper (Izumiura et al. in prep.).

The FIS was equipped with four detector arrays of different filters, N60, WIDE-S,WIDE-L, and N160, and their center wavelengths were $65,90,140$, and $160 \mu \mathrm{m}$, respectively. The FIS provided images in the four filters simultaneously. The arrays gave sampling intervals of $13^{\prime \prime}$ for N60 and WIDE-S and $22^{\prime \prime}$ for WIDE-L and N160 in the "cross-scan" direction that was perpendicular to the satellite's scanning ("in-scan") direction. This allowed images to be attained with the highest possible resolution, $\sim 40^{\prime \prime}$ for N60 and WIDE-S or $\sim 60^{\prime \prime}$ for WIDE-L and $N 160$, in the cross-scan direction determined by the detector pixel size and the telescope's point spread function.

We selected AOT (Astronomical Observation Template) FIS01, which was a slow scan mode for compact source photometry. This AOT performed two round trips around the target along the in-scan direction during one pointed observation that lasted $\sim 10 \mathrm{~min}$. A small offset was given in the cross-scan direction between the two round trips. The AOT, thus, provided four independent scanning images of the target by each detector array. The redundancy assured reliable maps of the target. We chose a scan speed of $15^{\prime \prime} \mathrm{s}^{-1}$ in the in-scan direction, a shift in the cross-scan direction of $70^{\prime \prime}$, and a reset interval of 1 second. This setting gave a sampling pitch in the in-scan direction of $\sim 15^{\prime \prime}$, which also allowed us to obtain images with the highest possible resolution in the in-scan direction. The spatial resolutions ( $F W H M$ s) achievable in-orbit by the AOT were $37^{\prime \prime}, 39^{\prime \prime}$, $58^{\prime \prime}$, and 61" for N60, WIDE-S, WIDE-L, and N160, respectively (Kawada et al. 2007). With the AOT and paremeter set we observed an area of $\sim 40^{\prime}$ in the in-scan direction by $\sim 10^{\prime}$ in the cross-scan direction during the pointed observation of U Hya.

The raw data were reduced by standard procedure using reduction software especially developed for the FIS data, FIS Slow-scan toolkit (slow-scan tool, version 20070914). It applies many kinds of corrections and rejections, such as flat fielding and high-energy particle events, to time series readouts from each individual pixel of the four detector arrays. Then it transforms the corrected readouts to the sky brightness at the sampling points on the sky and reconstructs far-infrared images from the corrected sky brightness at each sampling point on the sky measured by each detector pixel. We specified the following options, "TRRANS, /SL_RMV, /MEDIAN_FILTER, FITER_WIDTH $=200$, /ECLIPTIC" in the following slow-scan tool processing. With these options, corrections were made for the detector responsivity variation with time caused by the calibration lamp measurements (TRANS), removal of the stray light caused by the Earth shine reflected at the telescope baffle (SL_RMV), and background subtraction by median filtering (MEDIAN_FILTER) applied to the time series detector outputs

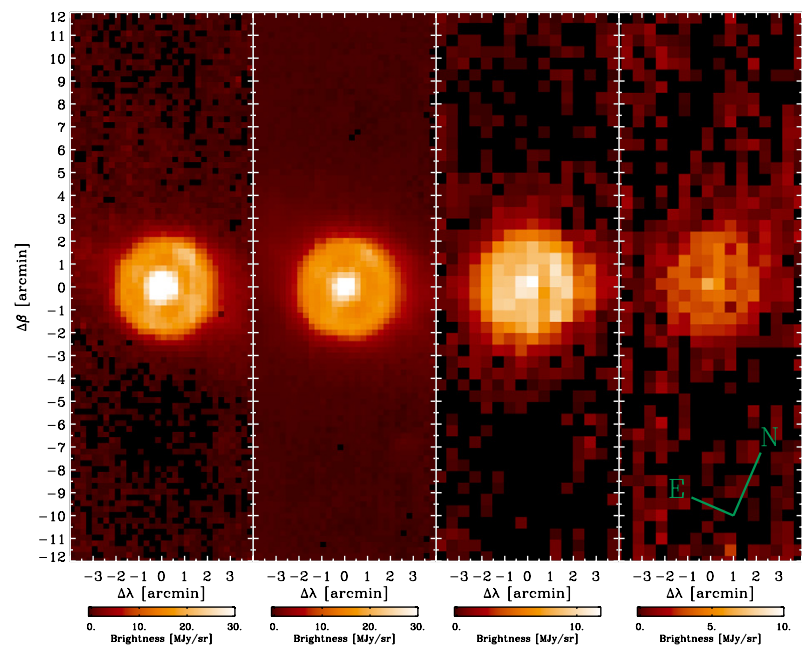

Fig. 1. From left to right the far-infared images of U Hya in $N 60(65 \mu \mathrm{m})$, $W I D E-S(90 \mu \mathrm{m}), W I D E-L(140 \mu \mathrm{m})$, and $N 160(160 \mu \mathrm{m})$ filters of the FIS on board AKARI, before color and extra intensity correction (see text). Pixel size is $15^{\prime \prime}$ in N60 and WIDE-S maps and $30^{\prime \prime}$ in WIDE$L$ and $N 160$ ones. The axes show the offsets with respect to the star. Ecliptic north is up and ecliptic east to the left. Equatorial north and east tilted by $-23^{\circ} .4$ with respect to the ecliptic coordinates are indicated in the bottom right corner.

with a filter width of FILTER_WIDTH in units of seconds of time. The sides of the resulting maps were aligned with the ecliptic coordinates by "/ECLIPTIC". The backgound level was, for example, about $9 \mathrm{MJy} \mathrm{sr}^{-1}$ in the WIDE-S band and $8 \mathrm{MJy} \mathrm{sr}^{-1}$ in the WIDE- $L$ band in the maps reconstructed in total power mode. We finally obtained the FIS images of U Hya in the four FIS bands, all with the sizes $\sim 10^{\prime} \times 40^{\prime}$, the longer sides of which were aligned in the ecliptic meridian.

\section{Results and analysis}

\subsection{Obtained images, flux densities, and corrections}

The images of U Hya produced by slow-scan tool in the four FIS bands are presented in Fig. 1. Here the central $8.5 \times 24^{\prime}$ part of each $\sim 10^{\prime} \times 40^{\prime}$ image is shown. The images reveal that there is a conspicuous, extended emission component surrounding the central point-like source. This source has an FWHMs of $\sim 40^{\prime \prime}$ in N60 and WIDE-S images and of $\sim 60^{\prime \prime}$ in WIDE-L and $N 160$ images. These $F W H M$ s are nearly identical to those of the point-source response function (hereafter referred to as PSRF) derived from in-orbit performance evaluation (Kawada et al. 2007). The extended emission is circular, and has a diameter of $\sim 4^{\prime}$, which agrees with the diameter of 3.5 found in the reconstructed high-resolution IRAS images (Waters et al. 1994), and is almost centered on the point-like component in each band.

The brightness of the extended part is about one fifth of the peak brightness at the central source in WIDE-S. The PSRF does not have such a strong extended component in any bands (see Kawada et al. 2007), indicating that the extended part is real. The sizes of the extended emission at the four bands are very similar, which also confirms that the emission is real. We also note that this extended emission is consistent with the excess emission seen in the IRAS survey scan data of $\mathrm{U}$ Hya in the 60 and $100 \mu \mathrm{m}$ bands (e.g., Young et al. 1993b). Our results thus confirm that U Hya has an extended component around the central point source. Furthermore, in the N60 and WIDE-S images the extended emission looks ring-like, showing a local minimum 


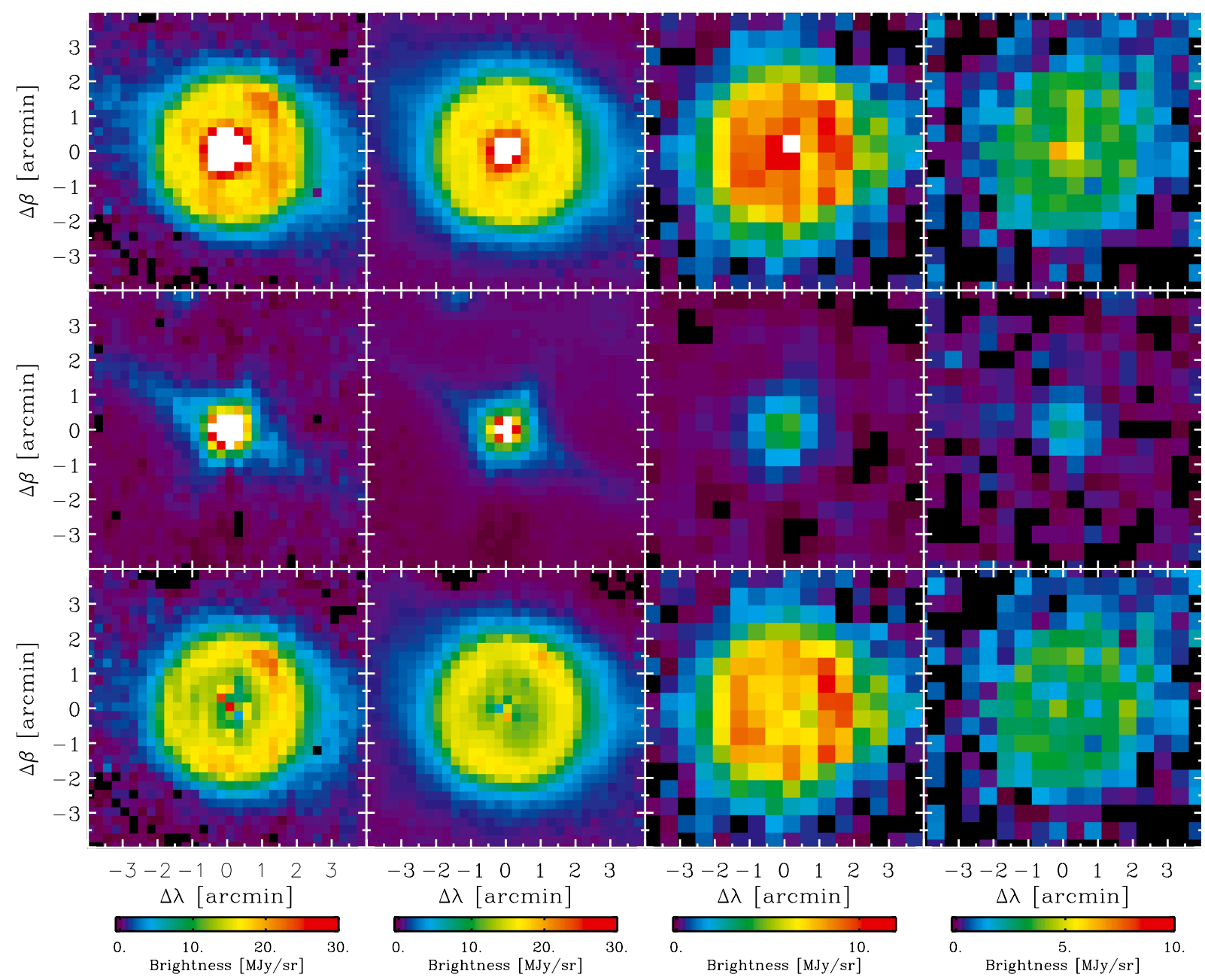

Fig. 2. Decomposition of (top row) the far-infrared image of U Hya at each FIS band shown in Fig. 1 into (middle row) a compact source and (bottom row) an extended shell. Each panel measures $8^{\prime} \times 8^{\prime}$. From left to right the columns shown are N60, WIDE-S, WIDE-L, and N160 images. Each color bar at the bottom is common to the column and shows the brightness scale before color and extra intensity corrections (see text) in units of $\mathrm{MJy} \mathrm{sr}^{-1}$. Ecliptic north is up and ecliptic east is to the left. Equatorial north and east are tilted by $-23^{\circ} 4$ as in Fig. 1 .

of the brightness at around $50^{\prime \prime}$ from the center, which is revealed for the first time by direct imaging in this star. The WIDE- $L$ and $N 160$ images show plateaus at the same distance from the central star, giving consistency with the N60 and WIDE-S data, though the lower spatial resolutions do not allow such local brightness minima to be revealed.

In Fig. 2 we show the decomposition of the original images of U Hya into the images of a central point-like source and an extended component. Here the central $7^{\prime} \times 7^{\prime}$ area is shown in each panel. We aligned and scaled an image of a point-source reference to the central point-like source in the U Hya image, then performed a point-source subtraction to obtain the shell image. We used images of early M-type giants observed in our AKARI MLHES mission program as point-source references ( $\beta$ Peg for $N 60$ and WIDE-S, $\beta$ Gru for WIDE-L and N160). The scaling factor for subtraction was iteratively determined so that the residual radial brightness profile would not have an apparent bump or dip at the center for each band. In other words, both the radial brightness profile and its derivative should be monotonically increasing functions of radius within $60^{\prime \prime}$. We found the scaling factor was determined reliably within 5\%. U Hya and the reference sources were observed with the same AOT parameters and their images were reconstructed with the same slowscan tool options. With this procedure, artifacts that affect the extended component, arising from the central point-like source through the PSRF of the FIS detectors, were eliminated in the resulting maps of the extended component.

After this decomposition we applied flux calibrations to the images of the central star and ring (the middle and bottom rows in Fig. 2) as described in Shirahata et al. (2009). We divided the middle and bottom images of N60,WIDE-S,WIDE-L, and $N 160$ by factors $0.603,0.607,0.56$, and 0.56 , respectively, except for the extended component in $N 160$, for which we used 0.277 consulting Shirahata et al. (2009). With this correction the flux densities of the central star were found to be 13.3, 7.60, 2.10, and $1.42 \mathrm{Jy}$ and the shell's flux densities over the entire shell to be 44.0, 44.7, 28.4, and 23.2 Jy, in N60, WIDE-S, WIDE-L, and $N 160$ filters, respectively, before color correction (Fig. 3).

We also applied color corrections (Shirahata et al. 2009) for the system responsivity characteristics across individual bands, defined by $S_{\text {true }}=S_{\text {obs }} / K$, where $S_{\text {true }}, S_{\text {obs }}$, and $K$ were the corrected flux density, observed flux density, and correction factor, respectively. The correction factors were 1.05, 1.41, 0.937, and 0.993 for N60, WIDE-S,WIDE-L, and N160, respectively, to the central source assuming its flux density $\propto v^{2}$, where $v$ is frequency. The factors for the extended component were calculated assuming its flux density is described by a modified black body at $45 \mathrm{~K}$, which was obtained from our preliminary analysis, with 
Table 1. Measured flux densities of U Hya.

\begin{tabular}{|c|c|c|c|c|}
\hline & $\begin{array}{l}N 60 \\
{[\mathrm{Jy}]}\end{array}$ & $\begin{array}{c}W I D E-S \\
{[\mathrm{Jy}]}\end{array}$ & $\begin{array}{c}\text { WIDE-L } \\
{[\mathrm{Jy}]}\end{array}$ & $\begin{array}{c}N 160 \\
{[\mathrm{Jy}]}\end{array}$ \\
\hline Compact & $12.7 \pm 0.4$ & $5.4 \pm 0.1$ & $2.2 \pm 0.1$ & $1.4 \pm 0.3$ \\
\hline Extended & $45.5 \pm 0.5$ & $43.4 \pm 0.2$ & $30.6 \pm 0.5$ & $23.0 \pm 1.5$ \\
\hline Systematic error ${ }^{a}$ & $20 \%$ & $20 \%$ & $10 \%$ & $50 \%$ \\
\hline & 9.90 & 7.4 & $(1.30)$ & $(0.77)$ \\
\hline Black body ${ }^{c}$ & 5.71 & 3.01 & 1.26 & 0.97 \\
\hline
\end{tabular}

Notes. ${ }^{(a)}$ Kawada et al.(2007). ${ }^{(b)}$ AKARI-FIS Bright Source Catalogue (Yamamura et al. 2010). ${ }^{(c)}$ For $2800 \mathrm{~K}, 2960 L_{\odot}$, and 162 pc (see text).

the power-law index of 1.1 , namely, $\propto v^{1.1} B_{v}(T)$, where $B_{v}(T)$ denotes the black body radiation at frequency $v$ and temperature $T$. We then found $0.967,1.028,0.926$, and 0.988 for N60, WIDE$S, W I D E-L$, and N160, respectively. This correction for a single temperature should be valid as far as we can assume that the dust grain temperature is determined by equilibrium with the stellar radiation field. The temperature difference inside the shell would be less than $\pm 5 \mathrm{~K}$ from $45 \mathrm{~K}$. Changing the temperature to $50 \mathrm{~K}$ gives the factor $+5 \%$ and $40 \mathrm{~K}$ gives $-5 \%$, which does not alter the results significantly.

By taking all the above corrections into account, we obtained the flux densities and their statistical errors of the central point-like source and the extended emission in the four bands (Table 1). Systematic errors in the absolute flux calibration from Kawada et al. (2007) are also given in Table 1. We also show the flux densities of U Hya in the AKARI-FIS bright source catalog (FIS BSC) based on the FIS all sky survey (Yamamura et al. 2010) for comparison. Apparently there are noticeable discrepancies between the flux densities we have obtained for the compact component and those from FIS BSC. We should note, however, that our measurements can carry $\sim 20 \%$ systematic errors. Similar systematic errors could also be associated to the FIS BSC values. Also, the flux density extraction scheme for FIS BSC is optimized for a point-like source, and its behavior is not well studied against a compact source overlaid on an extended, marginally resolved source. The FIS BSC values for $\mathrm{U}$ Hya should be taken as only a reference here. Verifying the reliability of an FIS BSC value for such a combination of a compact source on a partially resolved bright source is beyond the scope of this paper.

Our N60 measurements are found to be consistent with the past IRAS measurements: Young et al.(1993b) obtained $60 \mu \mathrm{m}$ flux densities of $12.5 \mathrm{Jy}$ and $48.3 \mathrm{Jy}$ for the central source and shell of U Hya, while Waters et al. (1994) found $18 \pm 3.5$ and $41 \pm 8 \mathrm{Jy}$ for the compact and compact plus extended components, respectively. In the bottom row of Table 1 "Black body" gives the expected flux densities at the FIS bands from a black body at a temperature of $2800 \mathrm{~K}$ with a luminosity of $2960 L_{\odot}$ located at 162 pc from the Sun (see Sect. 3.2.2). Dust emission from the unresolved inner circumstellar shell caused by the present-day mass loss of $1.2 \times 10^{-7} M_{\odot} \mathrm{yr}^{-1}$ can contribute to the flux densities of the compact component in Table 1, for example, by as much as a couple of Jy at $100 \mu \mathrm{m}$.

The resulting SEDs are displayed in Fig. 3. These spectral enegery distributions for the central source and the extended emission demonstrate that the former is hotter than the latter, indicating that the former is the star and the latter a cool dust shell. There should also be a contribution to the central point-like

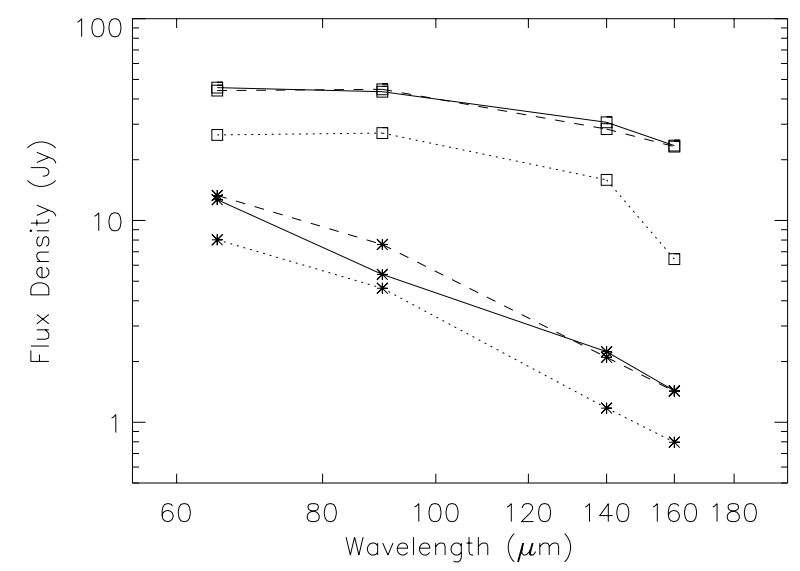

Fig. 3. Far-infrared spectral engergy distributions of the central pointlike source (asterisks) and the extended dust shell (squares). Dotted, dashed, and solid lines indicate raw, intensity corrected, and intensity and color corrected data, respectively (see text).

source from an unresolved dust shell that has been produced by the present-day mass loss of $1.2 \times 10^{-7} M_{\odot} \mathrm{yr}^{-1}$.

\subsection{Model analysis}

We analyzed the images of the dust shell (extended component) of U Hya with a simple model of a detached, spherical dust shell centered on the star. We note that the dust shell shows some faint structure, which is a question for our future analysis, and we ignore them in the following analysis.

For the present analysis, we re-determined the shell center. We minimized the scatter of data points at radii between $80^{\prime \prime}$ and $130^{\prime \prime}$ in each radial brightness profile by shifting the shell's center position one step at a time. We find that the shell center is slightly displaced from the central star position by $9^{\prime \prime}$ ( 0.6 pixels) to the right and $3^{\prime \prime}(0.2$ pixels) to the bottom on the $N 60$ and WIDE-S images in Figs. 1 and 2, where the pixel size is $15^{\prime \prime}$ and the uncertainty of the displacement is $\sim 2$.' 5 . This corresponds to 9.'5 distance between the two centers. The shell's center offset with respect to the star is at the position angle $(\mathrm{PA})=-85^{\circ} \pm$ $15^{\circ}$ in the equatorial coordinates. We neglect this small amount of displacement in the following analysis and discuss it later in Sect. 4.2.

\subsubsection{The model}

We fit the observed brightness distributions in the four bands simultaneously with a simple model of a spherical, detached dust shell (e.g., Izumiura et al. 1997). We assumed that dust grains in the shell are solely heated by and in radiative equilibrium with the stellar radiation and that the dust shell is optically thin at all wavelengths. Young et al. (1993b) find that the central AGB star is the dominant heating source of its extended dust shell out to a radius of 0.5 pc. Dust mass density $\rho(r)$ is assumed to vary as a power-law function of radial distance $r$ from the shell center with an index $\alpha$, namely, $\rho(r)=\rho_{0}\left(r / r_{0}\right)^{-\alpha}$, where $\rho_{0}$ is the dust mass density at the inner boundary of the shell, $r_{0}$. Dust grain cross section per unit mass of grain material (hereafter "dust absorptivity") $\kappa(\lambda)$ at a given wavelength $\lambda$ is chosen of a power-law function of $\lambda$ with an index $\beta$, namely, $\kappa(\lambda)=\kappa_{0}\left(\lambda / \lambda_{0}\right)^{-\beta}$, where $\kappa_{0}$ is the opacity at a reference wavelength $\lambda_{0}$. These assumptions 
Table 2. Convolving PSRF approximated by a double Gaussian.

\begin{tabular}{ccccccc}
\hline \hline $\begin{array}{c}\lambda \\
{[\mu \mathrm{m}]}\end{array}$ & $\begin{array}{c}\theta_{1} \\
{[\operatorname{arcsec}]}\end{array}$ & hght1 & wght1 & $\begin{array}{c}\theta_{2} \\
{[\operatorname{arcsec}]}\end{array}$ & hght2 & wght2 \\
\hline 65 & 32.05 & 0.790 & 0.392 & 77.5 & 0.210 & 0.608 \\
90 & 30.17 & 0.797 & 0.325 & 86.0 & 0.203 & 0.675 \\
140 & 40.85 & 0.760 & 0.272 & 119.0 & 0.240 & 0.728 \\
160 & 38.23 & 0.799 & 0.285 & 120.6 & 0.201 & 0.715 \\
\hline
\end{tabular}

also allow us to describe the dust temperature distribution $T_{\mathrm{d}}(r)$ in the shell as a power-law function of distance from the shell centrer: $T_{\mathrm{d}}(r)=T_{0}\left(r / r_{0}\right)^{-2 /(4+\beta)}$ (see Sopka et al. 1988), where $T_{0}$ is the dust temperature at $r_{0}$.

In our analysis, we convolved the model brightness distribution with a model PSRF described by two circularly symmetric, concentric Gaussians with different heights and FWHMs given in Table 2 (see Shirahata et al. 2009). The second, third, and fourth columns give the $F W H M$, relative height, and relative weight, respectively, of the first narrower component and the fifth, sixth, and seventh those of the second broader component. Then, the brightness at the center of each pixel position on the FIS image was calculated from the convolved model image.

\subsubsection{Fixed parameters}

We adopted $162 \mathrm{pc}$ as the distance to U Hya based on the revised Hipparcos parallax of 6.18 mas (van Leeuwen 2007). We assumed the central star is a blackbody source. We set its temperature at $2800 \mathrm{~K}$, while Tsuji (1981) gave $2825 \mathrm{~K}$ and Groenewegen et al. (1992) did $2730 \mathrm{~K}$. We find that the luminosity is $2960 L_{\odot}$ based on the observed bolometric flux given by Groenewegen et al.(1992) and the Hipparcos distance of 162 pc. For calculating quantities related to the dust mass, we adopted a dust absorptivity at $100 \mu \mathrm{m}$ (hereafter referred to as $\kappa_{100}$ ) of $25 \mathrm{~cm}^{2} \mathrm{~g}^{-1}$ (Hildebrand 1983), while other higher values are also found in the literature (for example see Draine 1981; Nakada 1988; Groenewegen et al. 1998). For mass-loss rate calculation we assumed an outflow velocity of $15.0 \mathrm{~km} \mathrm{~s}^{-1}$. These uncertainties can be treated properly in the following.

\subsubsection{Free parameters}

With the above assumptions and fixed paremeters of the model there remain five free paramters, $R_{\mathrm{in}}, \Delta R, \alpha, \beta$, and $\rho_{0}$ to calculate the model brightness profile at each FIS band, where $R_{\text {in }}$ is the shell inner boundary radius and $\Delta R$ the shell thickness, and $T_{0}$ was calculated from the effective temperature and luminosity of the central source, $\beta$, and $R_{\text {in }}$.

\subsubsection{Results of model analysis}

By changing the five free parameters one at a time, we searched the five-dimensional parameter space for the minimum reduced $\chi^{2}=\Sigma_{r_{1}<r<r_{2}}\left[\left(B_{\text {obs }}-B_{\text {model }}\right) / E_{\text {obs }}\right]^{2} /(n-5)$ over a specific annular area (of $r_{1}<r<r_{2}$ ) in the four images together. $B_{\text {obs }}, B_{\text {model }}$, and $E_{\mathrm{obs}}$ are observed brightness, model brightness, and error estimate at each pixel, respectively, and $n$ is the number of pixels used to calculate the $\chi^{2}$. The steps were $7.0 \times 10^{15} \mathrm{~cm}\left(2^{\prime \prime} .88\right)$, $7.3 \times 10^{15} \mathrm{~cm}, 2.5 \times 10^{-25} \mathrm{~g} \mathrm{~cm}^{-3}, 0.5$, and 0.05 for $R_{\mathrm{in}}, \Delta R, \rho_{0}$, $\alpha$, and $\beta$, respectively. We used three different annular regions of fitting, $40^{\prime \prime}-160^{\prime \prime}, 50^{\prime \prime}-150^{\prime \prime}$, and $60^{\prime \prime}-140^{\prime \prime}$, to explore their effects on the fitting results. The best-fit models for the three annular areas are shown in Fig. 4. We find that the simple dustshell model reproduces the observed brightness distribution well in each band, in particular, at WIDE-S where the highest $S / N$ is achieved.

We summarize the best-fit parameters for the three annular areas in the first three rows of Table 3 . In addition to the five free parameters searched, we give a half-mass width of the shell $\Delta R_{\mathrm{hm}}$, within which one half of the total dust mass is contained, measured from $R_{\text {in }}$ as a measure of the effective shell thickness in the fourth column. The eighth, ninth, and tenth columns give the dust temperature at $R_{\text {in }}$, the dust mass in the shell, and the dust loss rate at $R_{\text {in }}$, respectively.

Both $R_{\text {in }}$ and $\beta$ were constrained reliably in the fitting, and $R_{\text {in }}$ was predominantly determined by the position of the brightness peak and $\beta$ by the multi-band observations. The inner radius of 107" confirmed the result from the high-resolution IRAS image of 105" (Waters et al. 1994). Young et al. (1993a) also obtained compatible inner and outer radii of 1 '.3 and 2'.9 for the dust shell of U Hya assuming an elliptical dust shell of uniform density and temperature. We obtained 1.15 for $\beta$, while Jura (1986) derived 1.1 as a representative value for carbon grains in many carbon stars, suggesting amorphous carbon rather than graphite for the state of carbon in outflowing gas.

The remaining three parameters $\Delta R, \rho_{0}$, and $\alpha$ also converged in similar sets of values for the three different annular fitting areas. The high $\alpha$ values imply that the dust shell is a thin wall of dust concentrated in a physically narrow region. The behavior is depicted in Fig. 5 as the underlying models described by the parameters. We should, however, note that the three parameters are less well constrained than are $R_{\text {in }}$ and $\beta$ for the following two reasons. The first one is that $\Delta R$, hence $\alpha$, tend to become too large because the model PSRF has a deficiency in the outermost part $\left(r>\sim 50^{\prime \prime}\right)$ compared with the real PSRF (see Shirahata et al. 2009). The second one is that the three parameters are entangled because the shell's effective width is smaller than the spatial resolutions of the FIS instrument, which we have learned from the present analysis, as indicated by the small $\Delta R_{\mathrm{hm}}$. Such a narrow width allows various combinations of $\rho_{0}$ and $\alpha$ to give similarly small $\chi^{2}$. This is the most evident for the smallest annular fitting area of $60^{\prime \prime}-140^{\prime \prime}$ (bottom five rows of Table 3 and Figs. 6 and 7), for which the deficiency of the model PSRF has the least influence on the fitting results among the three fitting areas.

Resulting dust mass $\left(M_{\text {dust }}\right)$ stays rather constant, as is evident from Fig. 7 and Table 3, because the dust mass is approximately proportional to the amount of observed emission under the employed dust temperature law that varies slowly with distance from the central star. Dust temperature in the shell is between $40 \mathrm{~K}$ and $50 \mathrm{~K}$ for the best-fit models, consistent with the observed mean brightness ratio $B(65 \mu \mathrm{m}) / B(90 \mu \mathrm{m}) \sim 1$, which corresponds to $49 \mathrm{~K}$ for the modified black body with the power-law index of 1.1. The derived dust mass is a robust number except for the uncertainty in the magnitude of $\kappa_{100}$.

In the above analysis we found that we could significantly improve the simultaneous fitting of a model to the four band data if we decreased the $N 60$ data or increased the WIDE-S data by $10 \%$ ( $\chi^{2}$ from 6.01 to 4.88 for the range $60^{\prime \prime}-140^{\prime \prime}$ ). This scaling may be justified by the spectral energy distributions that show kinks at $90 \mu \mathrm{m}$ as shown in Fig. 2. The flux densities of both point-like and extended components seem to have a deficiency of $10-20 \%$ at $W I D E-S(90 \mu \mathrm{m})$ band if we postulate smooth spectral energy distributions. Thus, we repeated searching the parameter space for the best-fit models for the same images but with the WIDE-S data arbitrary scaled-up by $10 \%$. 

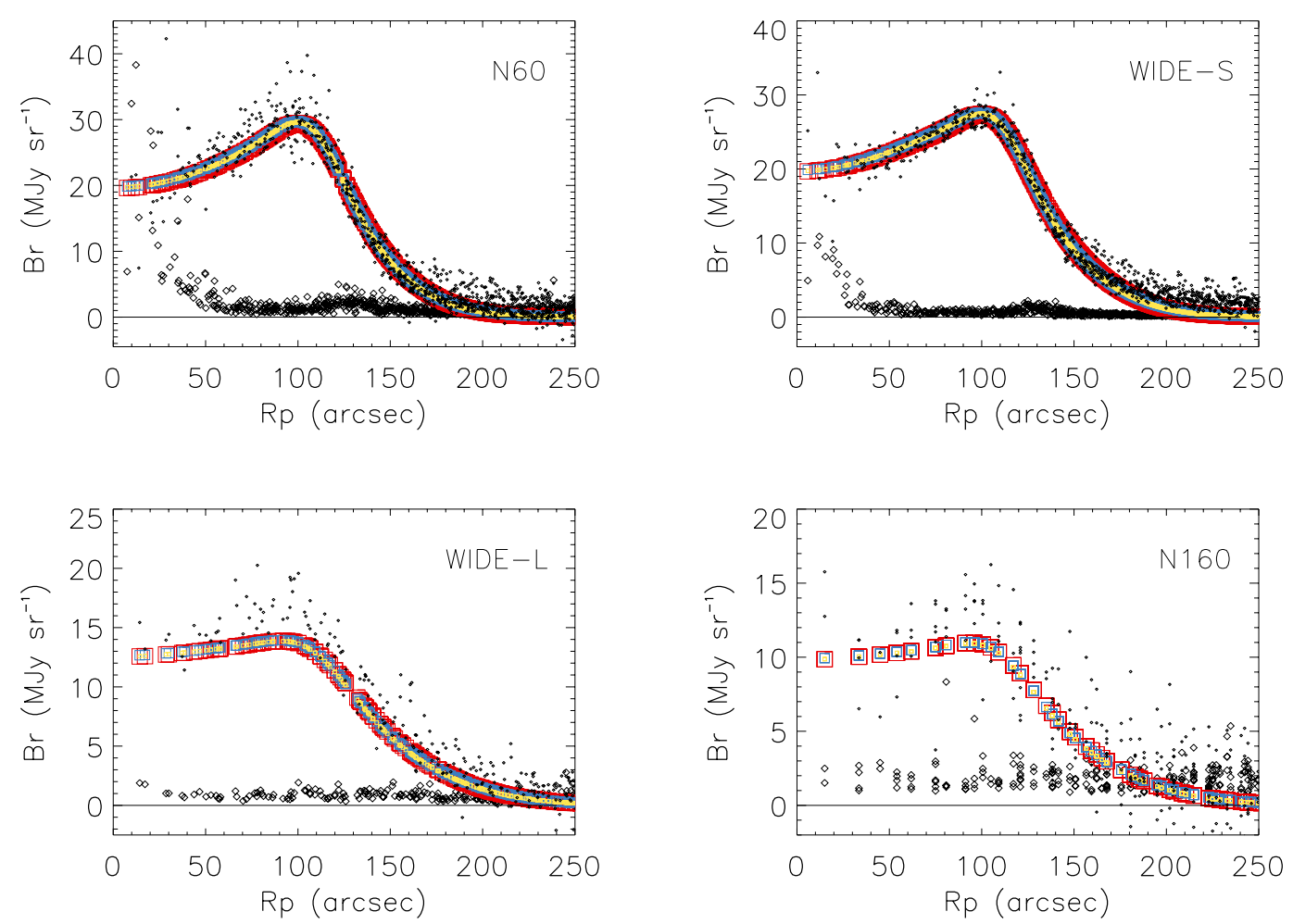

Fig. 4. Observed and model radial brightness profiles of the dust shell of U Hya in N60, WIDE-S, WIDE-L, and N160 bands. Ordinate and abscissa are brightness in units of $\mathrm{MJy} \mathrm{sr}^{-1}$ and apparent distance from the center projected on the sky in arcseconds, respectively. In each panel, dots indicate observations extracted from the bottom row images of Fig. 2 with all corrections taken into account. Open diamonds show statistical error estimates. Red, cyan, and yellow open squares display the best-fit models given in Table 3 for the annular areas of $40^{\prime \prime}-160^{\prime \prime}, 50^{\prime \prime}-150^{\prime \prime}$, and $60^{\prime \prime}-140^{\prime \prime}$, respectively.

Table 3. Best-fit parameters of the model dust shell for different annular fitting areas.

\begin{tabular}{|c|c|c|c|c|c|c|c|c|c|c|c|}
\hline $\begin{array}{l}\text { Fit range } \\
{[\operatorname{arcsec}]}\end{array}$ & $\begin{array}{c}R_{\text {in }} \\
{\left[10^{17} \mathrm{~cm}\left({ }^{\prime \prime}\right)\right]}\end{array}$ & $\begin{array}{c}\Delta R \\
{\left[10^{17} \mathrm{~cm}\left({ }^{\prime \prime}\right)\right]}\end{array}$ & $\begin{array}{l}\Delta R_{\mathrm{hm}} \\
{\left[{ }^{\prime \prime}\right]}\end{array}$ & $\begin{array}{c}\rho_{0}{ }^{a} \\
{\left[\mathrm{~g} \mathrm{~cm}^{-3}\right]}\end{array}$ & $\alpha$ & $\beta$ & $\begin{array}{c}T_{\mathrm{d}}\left(R_{\mathrm{in}}\right) \\
{[\mathrm{K}]}\end{array}$ & $\begin{array}{l}M_{\text {dust }}^{a} \\
{\left[M_{\odot}\right]}\end{array}$ & $\begin{array}{c}\dot{M}_{\mathrm{dust}}^{a, b} \\
{\left[M_{\odot} \mathrm{yr}^{-1}\right]}\end{array}$ & $x^{2}$ & $n-5$ \\
\hline $40-160$ & $2.59(107)$ & $2.09(86)$ & 19.0 & $3.75 \times 10^{-24}$ & 6.5 & 1.15 & 49.7 & $1.03 \times 10^{-4}$ & $7.5 \times 10^{-8}$ & 6.20 & 831 \\
\hline $50-150$ & $2.59(107)$ & $2.73(113)$ & 18.5 & $4.00 \times 10^{-24}$ & 7.0 & 1.15 & 49.7 & $1.04 \times 10^{-4}$ & $8.1 \times 10^{-8}$ & 6.39 & 699 \\
\hline $60-140$ & $2.59(107)$ & $2.23(92)$ & 17.7 & $4.00 \times 10^{-24}$ & 7.0 & 1.15 & 49.7 & $1.01 \times 10^{-4}$ & $8.1 \times 10^{-8}$ & 5.93 & 550 \\
\hline \multirow[t]{5}{*}{$60-140$} & $2.45(101)$ & $0.79(33)$ & 22.8 & $1.00 \times 10^{-24}$ & -4.5 & 1.15 & 50.8 & $0.89 \times 10^{-4}$ & $1.8 \times 10^{-8}$ & 6.08 & 550 \\
\hline & $2.45(101)$ & $0.87(36)$ & 23.4 & $1.25 \times 10^{-24}$ & -2.5 & 1.15 & 50.8 & $0.90 \times 10^{-4}$ & $2.3 \times 10^{-8}$ & 5.99 & 550 \\
\hline & $2.52(104)$ & $0.87(36)$ & 21.0 & $1.75 \times 10^{-24}$ & -0.5 & 1.15 & 50.3 & $0.91 \times 10^{-4}$ & $3.3 \times 10^{-8}$ & 6.02 & 550 \\
\hline & $2.52(104)$ & $0.94(39)$ & 20.1 & $2.25 \times 10^{-24}$ & 1.5 & 1.15 & 50.3 & $0.92 \times 10^{-4}$ & $4.4 \times 10^{-8}$ & 6.01 & 550 \\
\hline & 2.59 (107) & $1.01(42)$ & 16.6 & $3.25 \times 10^{-24}$ & 4.5 & 1.15 & 49.7 & $0.93 \times 10^{-4}$ & $6.5 \times 10^{-8}$ & 5.99 & 550 \\
\hline
\end{tabular}

Notes. ${ }^{(a)} \kappa_{100}=25 \mathrm{~cm}^{2} \mathrm{~g}^{-1}$ is assumed in this and later tables. ${ }^{(b)}$ At $R_{\mathrm{in}}$ and $v_{\mathrm{e}}=15.0 \mathrm{~km} \mathrm{~s}^{-1}$ are assumed in this and later tables.

The results are given in Table 4 , which are similar to those obtained without modification to WIDE-S data. They can be used to evaluate the unertainties of the quantities given in the tables, since the differences between Tables 3 and 4 come from the $10 \%$ change in the WIDE-S data. This time we have obtained $\beta=1.10$, the same value as in Jura (1986).

\subsubsection{Mass and mass-loss rate in the shell}

From the model analysis, the dust mass in the shell is confined well within $20 \%$ of $1.1 \times 10^{-4}\left(\kappa_{100} / 25\right)^{-1} M_{\odot}$. The obtained dust mass on the order of $10^{-4} M_{\odot}$ is comparable to what is estimated from optical observations $\left(\sim 2 \times 10^{-5} M_{\odot}\right.$;
Izumiura et al. 2007), given the uncertainty of $\kappa_{100}$. This gives a total mass of $\sim 10^{-2}\left(\kappa_{100} / 25\right)^{-1}\left(f_{\mathrm{gd}} / 100\right) M_{\odot}$, where $f_{\mathrm{gd}}$ is the gas-to-dust mass ratio, which is considered to be in the range of 200-800 (Jura 1986; Kerschbaum et al. 1996; Groenewegen et al. 1998). The last two terms of the total mass act to cancel each other out, and their product is almost unity for the case of carbon stars (for example see Jura 1986; Groenewegen et al. 1998). No direct estimate of the total gas mass is available in the literature for the extended shell of U Hya. Gérard \& Le Bertre (2006) detected the $21 \mathrm{~cm}$ atomic hydrogen emission toward $\mathrm{U}$ Hya. The diameter and mass of the $\mathrm{H}$ I envelope they find are $32^{\prime}$ and $0.0055 M_{\odot}$, respectively. The size suggests that it is not a direct counterpart of the observed dust shell of U Hya, though its 

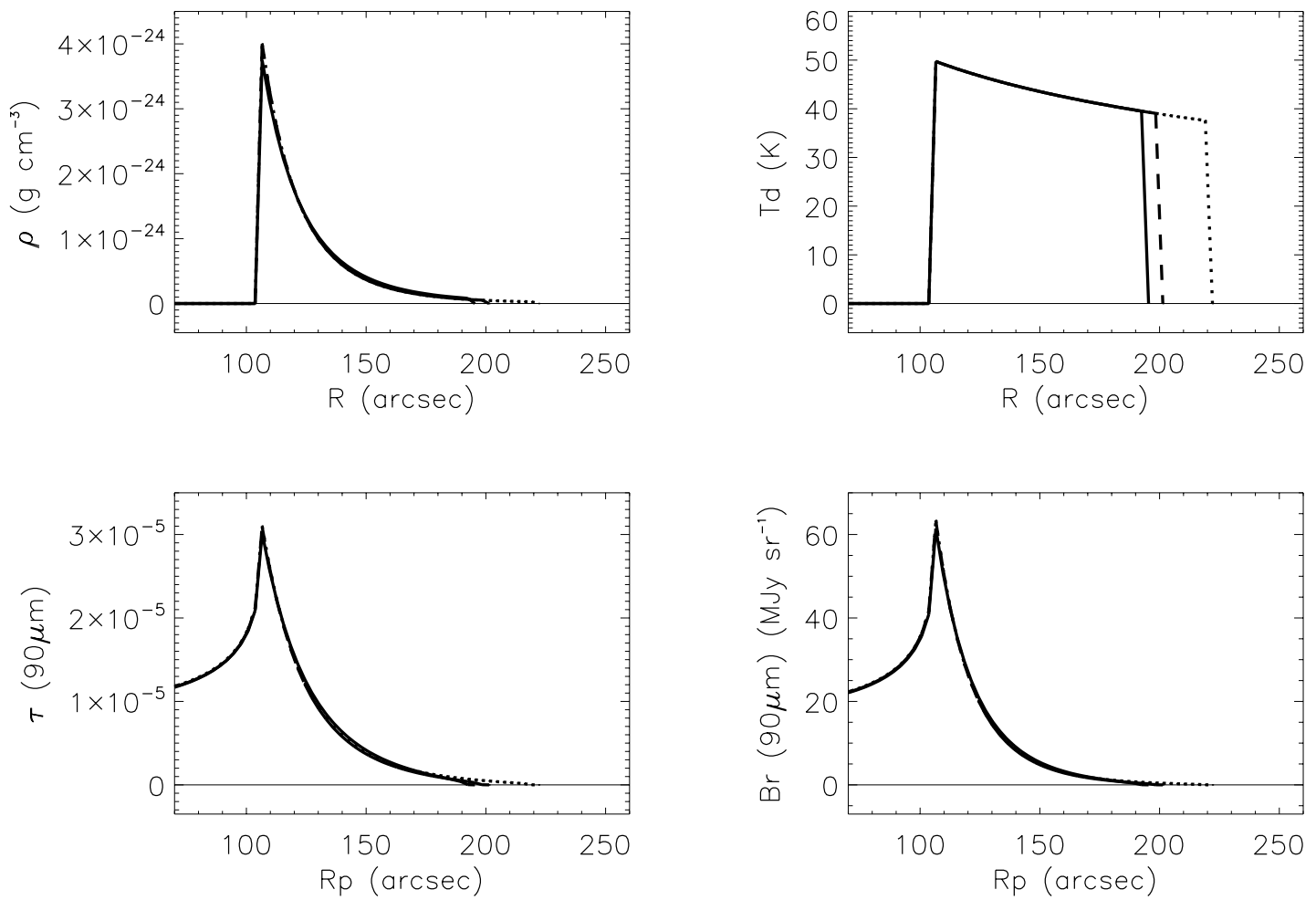

Fig. 5. (Upper panels) Density and temperature distributions in the model shells shown in Fig. 4, (lower panels) resulting optical depth and brightness profiles of the models in the WIDE-S band before being convolved with the point source response function given in Table 2 . Abscissa of the upper panels is the apparent radial distance from the center. Solid, dotted, and dashed lines indicate the results for annular areas of $40^{\prime \prime}-160^{\prime \prime}$, $50^{\prime \prime}-150^{\prime \prime}$, and $60^{\prime \prime}-140^{\prime \prime}$, respectively. The lines mostly overlap one another and are hardly seen separately.
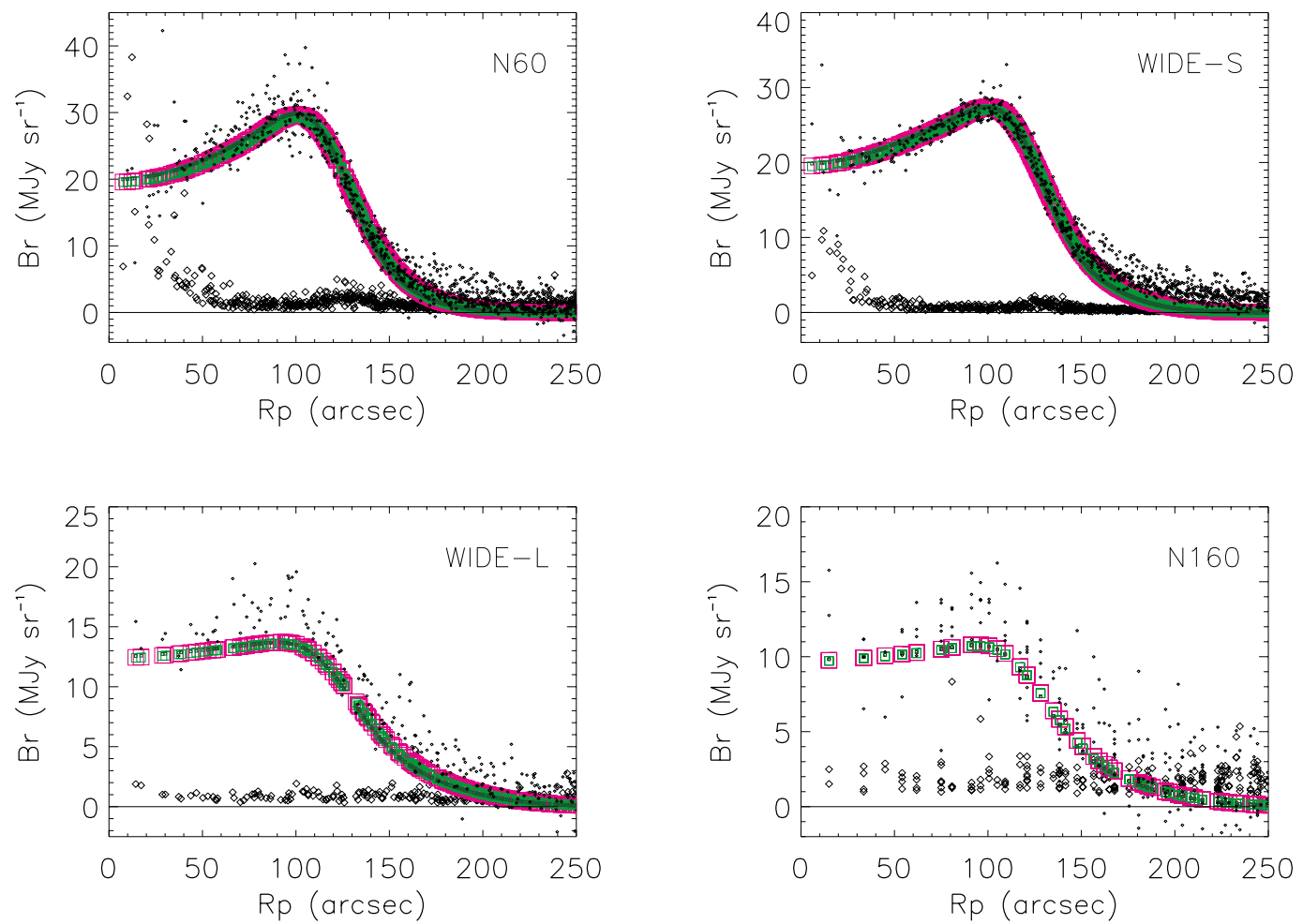

Fig. 6. Same as in Fig. 4 but for models with $\alpha=-4.5,-0.5$, and 4.5 shown as small dark green, medium-sized green, and large magenta squares, respectively, for the annular area of $60^{\prime \prime}-140^{\prime \prime}$ (see Table 3). 

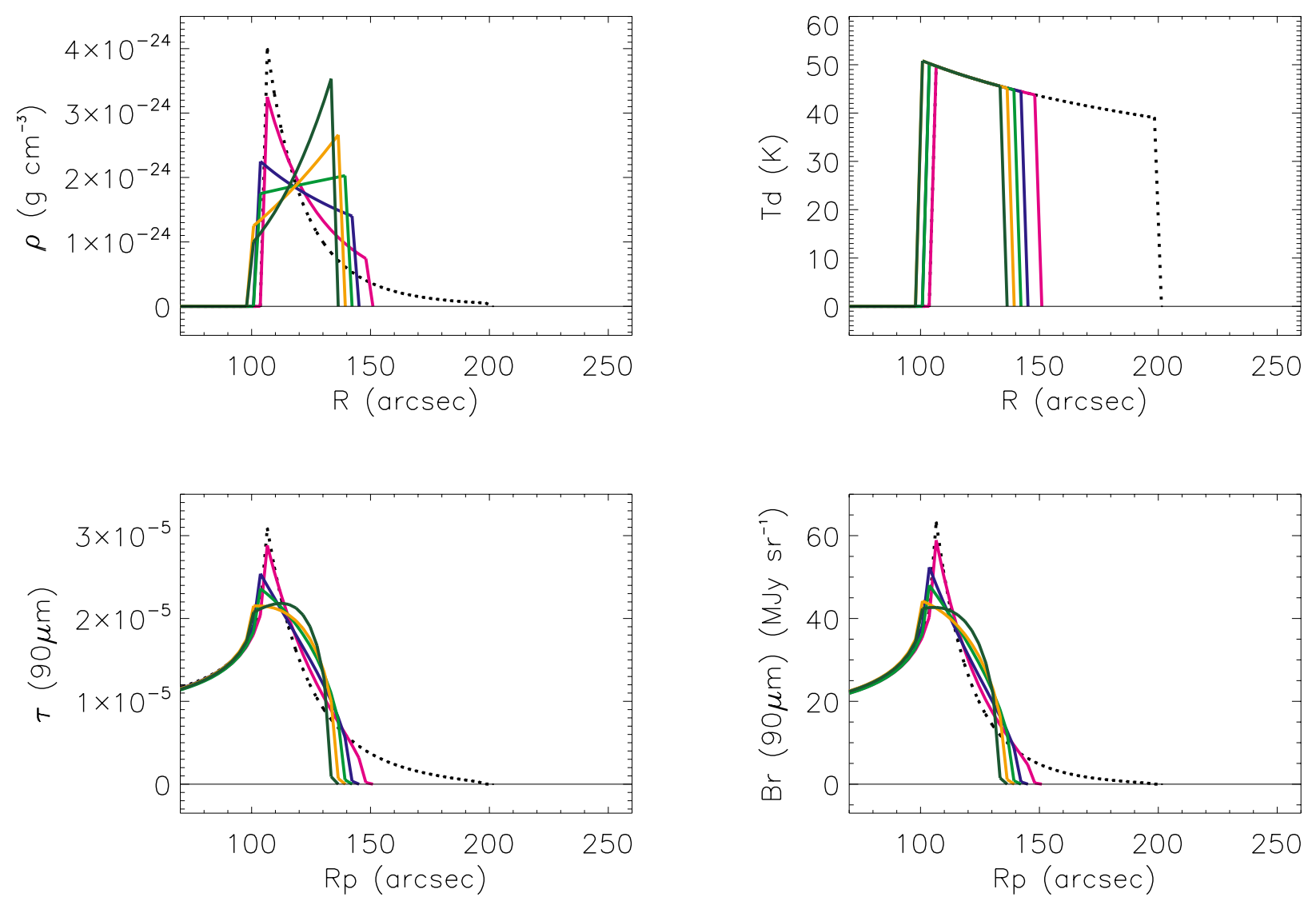

Fig. 7. Same as in Fig. 5 but for models that give similarly small $\chi^{2}$ for the annular area of $60^{\prime \prime}-140^{\prime \prime}$ (see Table 3), where dark green, orange, green, blue, and magenta lines for $\alpha=-4.5,-2.5,-0.5,1.5$, and 4.5 , respectively. The model for $60^{\prime \prime}-140^{\prime \prime}$ with $\alpha=7.0$ in Table 3 is also shown as a dotted black line for comparison.

mass seems compatible with both optical and FIR observations. Young et al. (1993a) estimated the total mass in the extended circumstellar shell to be $4 \times 10^{-3} M_{\odot}$ by assuming that the shell is composed of a pile up of continuous mass-loss wind retained by the interstellar matter. The total mass should be reduced by a factor 1.8 since they assumed $290 \mathrm{pc}$ instead of $162 \mathrm{pc}$ for the distance. Then, their total mass seems insufficient, while their estimates of the FIR emission agree with our FIS observations.

We also find that a half of the dust mass is confined in a relatively narrow region within a width of $16-23^{\prime \prime}$ or $(3.9-5.6) \times 10^{16} \mathrm{~cm}$ from the inner boundary of the shell, in any case, which is an important constraint on the detached dust shell of U Hya. The dust mass-loss rate at $R_{\text {in }}$ is $(1.8-9.6) \times$ $10^{-8}\left(v_{\mathrm{e}} / 15\right)\left(\kappa_{100} / 25\right)^{-1} M_{\odot} \mathrm{yr}^{-1}$, where $v_{\mathrm{e}}$ is the expansion velocity at the inner boundary in units of $\mathrm{km} \mathrm{s}^{-1}$. This gives a total mass-loss rate of $(1.8-9.6) \times 10^{-6}\left(v_{\mathrm{e}} / 15\right)\left(\kappa_{100} / 25\right)^{-1}$ $\left(f_{\mathrm{gd}} / 100\right) M_{\odot} \mathrm{yr}^{-1}$, which is compatible with $5 \times 10^{-6} M_{\odot} \mathrm{yr}^{-1}$ given by Waters et al. (1994).

We also find that the dust density at $118^{\prime \prime}$ is robust: $\rho\left(118^{\prime \prime}\right)=1.8(2.4) \times 10^{-24}\left(\kappa_{100} / 25\right)^{-1} \mathrm{~g} \mathrm{~cm}^{-3}$ as can be seen in Fig. 7 for the case of power-law dust density distribution. The number in the parentheses is for the case of the modified WIDE$S$ data (see Sect. 3.2.4). We note that a triangular and a Gaussian shape dust density distributions would also be viable for the observed images, although we have not examined either case yet.

\section{Discussion}

\subsection{Origin of the detached shell}

Both far-infrared excess emission and far-infrared extended emission have been recognized in AGB stars since the early stage of IRAS studies of evolved stars. Such far-infrared excess emission suggests there is a cold detached dust shell. RowanRobinson et al. (1986) concluded that the $100 \mu \mathrm{m}$ excess emission in evolved stars came from a detached shell located at the region where the mass-loss wind was running into the interstellar matter. However, van der Veen \& Habing (1988) suggested a discontinuity in the mass-loss process during the AGB evolution as a viable explanation for the excess FIR emission, invoking a possible commitment of a thermal pulse.

Willems \& de Jong (1988) successfully modeled the distribution of carbon stars on the IRAS 12-25-60 $\mu \mathrm{m}$ two-color diagram by introducing a scenario that an interruption of the preceding high mass-loss phase by a thermal pulse had caused the detached dust shells in carbon stars that were bright in the optical. They derived the mass-loss rate was higher by an order of magnitude in the previous high mass-loss phase than in the post low mass-loss phase. While this scenario seems attractive, a unanimous conclusion has not been drawn yet for the actual origin and formation mechanism of the detached dust shells in carbon stars. In particular, other new scenarios have emerged (Steffen \& Schönberner 2000; Libert et al. 2007) based 
Table 4. Best-fit parameters of the model dust shell with modifed WIDE-S data for different annular fitting areas.

\begin{tabular}{|c|c|c|c|c|c|c|c|c|c|c|c|}
\hline $\begin{array}{l}\text { Fit range } \\
\text { [arcsec] }\end{array}$ & $\begin{array}{c}R_{\text {in }} \\
{\left[10^{17} \mathrm{~cm}\left({ }^{\prime \prime}\right)\right]}\end{array}$ & $\begin{array}{c}\Delta R \\
{\left[10^{17} \mathrm{~cm}\left({ }^{\prime \prime}\right)\right]}\end{array}$ & $\begin{array}{l}\Delta R_{\mathrm{hm}} \\
{\left[{ }^{\prime \prime}\right]}\end{array}$ & $\begin{array}{c}\rho_{0} \\
{\left[\mathrm{~g} \mathrm{~cm}^{-3}\right]}\end{array}$ & $\alpha$ & $\beta$ & $\begin{array}{c}T_{\mathrm{d}}\left(R_{\text {in }}\right) \\
{[\mathrm{K}]}\end{array}$ & $\begin{array}{l}M_{\text {dust }} \\
{\left[M_{\odot}\right]}\end{array}$ & $\begin{array}{c}\dot{M}_{\text {dust }} \\
{\left[M_{\odot} \mathrm{yr}^{-1}\right]}\end{array}$ & $\chi^{2}$ & $n-5$ \\
\hline $40-160$ & $2.59(107)$ & $2.09(86)$ & 19.0 & $4.75 \times 10^{-24}$ & 6.5 & 1.10 & 47.8 & $1.30 \times 10^{-4}$ & $9.6 \times 10^{-8}$ & 5.32 & 831 \\
\hline $50-150$ & 2.59 (107) & $1.94(80)$ & 18.6 & $4.75 \times 10^{-24}$ & 6.5 & 1.10 & 47.8 & $1.28 \times 10^{-4}$ & $9.6 \times 10^{-8}$ & 5.38 & 699 \\
\hline $60-140$ & 2.59 (107) & $1.15(58)$ & 15.5 & $4.75 \times 10^{-24}$ & 6.0 & 1.10 & 47.8 & $1.16 \times 10^{-4}$ & $9.6 \times 10^{-8}$ & 4.67 & 550 \\
\hline \multirow[t]{4}{*}{$60-140$} & $2.45(101)$ & $0.79(33)$ & 22.8 & $1.25 \times 10^{-24}$ & -4.5 & 1.10 & 48.8 & $1.11 \times 10^{-4}$ & $1.8 \times 10^{-8}$ & 4.79 & 550 \\
\hline & $2.52(104)$ & $0.79(33)$ & 19.6 & $2.25 \times 10^{-24}$ & -1.0 & 1.10 & 48.3 & $1.13 \times 10^{-4}$ & $4.4 \times 10^{-8}$ & 4.73 & 550 \\
\hline & $2.52(104)$ & $0.87(36)$ & 19.1 & $2.75 \times 10^{-24}$ & 1.0 & 1.10 & 48.3 & $1.12 \times 10^{-4}$ & $5.3 \times 10^{-8}$ & 4.77 & 550 \\
\hline & $2.52(104)$ & $1.08(45)$ & 20.3 & $3.25 \times 10^{-24}$ & 3.0 & 1.10 & 48.3 & $1.17 \times 10^{-4}$ & $6.2 \times 10^{-8}$ & 4.73 & 550 \\
\hline
\end{tabular}

on the discoveries of extended, detached shells of gas (Olofsson et al. 1988, 1990, 1993) and dust (Waters et al. 1994; Izumiura et al. 1996, 1997) in a handful of carbon stars that show excess far-infrared emission and on the detection of huge $\mathrm{H}$ I gas envelopes in both oxygen-rich and carbon-rich AGB stars (Gérard $\&$ Le Bertre 2006). Those detections have also stimulated hydrodynamical simulations of dusty wind from thermally pulsing AGB stars. We consider the formation mechanism of the dust shell of U Hya by referring to these scenarios in the following.

\subsubsection{Thermal pulse and two-wind interaction}

Steffen et al. (1998) and Steffen \& Schönberner (2000, hereafter SS00) simulated the time-development of dusty circumstellar shells of gas and dust in the final stages of the AGB evolution. They suggest that a typical helium-shell flash can trigger a mass-loss eruption, which in turn gives rise to a short period of a high outflow velocity and a subsequent two-wind interaction (cf. Kwok et al. 1978) that produces a thin detached gas shell. They also show that a sharp decline in the mass-loss rate will follow a thermal pulse, which generates a detached dust shell. Mattsson et al. (2007) have computed the development of detached circumstellar shells using their detailed radiation hydrodynamical models including dust formation of the atmospheric layers and wind accerelation region around TP-AGB stars. They find that the formation of a slow and a fast wind combined with an eruptive mass loss, which is associated with the fast wind as a result of a thermal pulse, is critical for the production of a thin gas shell. In other words, both the two wind interaction and the eruptive mass loss are necessary for the co-existence of thin detached gas and dust shells.

Some of the carbon stars with the far-infared excess emission are also known to show molecular envelopes with detached structure in $\mathrm{mm}$-wave $\mathrm{CO}$ rotational lines (Olofsson et al. 1996, 2000; Schöier et al. 2005, and references therein). The co-existence of the detached shells of gas and dust in those carbon stars seem to match the scenario well that an eruptive mass loss caused by a thermal pulse triggers the two-wind interaction (SS00; Mattsson et al. 2007). Schöier et al. (2005) concluded from their observations of the thin detached CO gas shells that a two-wind interaction exerted by an eruptive rapid mass loss is the cause of the molecular gas shells. They find that the mass-loss eruptions occurred at rates two orders of magnitude higher than those of the preceding phases $\left(10^{-5} M_{\odot} \mathrm{yr}^{-1}\right.$ and $10^{-7} M_{\odot} \mathrm{yr}^{-1}$, respectively) and their durations were as short as a few hundred years. They further suggest a connection between a mass-loss eruption and an He shell flash.
There are three carbon stars that possess detached dust shells show up in the FIR but do not have corresponding thin molecular gas shells. They are U Hya (Waters et al. 1994; present paper), U Ant (Izumiura et al. 1997), and Y CVn (Izumiura et al. 1996). As for U Ant we note that the inner shell of its double dust shell has a detached thin CO shell counterpart (Olofsson et al. 1990) and is also observed in optical light scattered by dust grains (Maercker et al. 2010). We consider here the outer dust shell of its double dust shell and refer to it as "U Ant(outer)". It is most likely that $\mathrm{U}$ Hya experienced a thermal pulse in the last $\sim 10^{5}$ years since it shows ${ }^{99} \mathrm{Tc}$ in its photosphere (Peery 1971), presumably due to a recent episode of the third dredge-up exerted by a thermal pulse. Though it is not proved yet, the double dust shell of U Ant also favors thermal pulse events (Izumiura et al. 1997; Schöier et al. 2005). No strong evidence is known for or against a thermal pulse event in Y CVn. SSO0 argue that only an abrupt reduction of mass loss is enough for the formation of a detached dust shell. They also note that all thermal pulses should produce such a shell, but not all of them will have the right properties for producing a pronounced thin molecular shell. Survival of a molecular shell at a large distance from the central star strongly depends on the degree of photodissociation by the interstellar radiation field. The dust shells of U Hya, U Ant(outer), and Y CVn may be formed through the mechanism proposed by SS00 and Mattsson et al. (2007). Photodissociation may account for the absence of detached CO shells associated with their dust shells (Meixner et al. 1988).

Other differences among detached dust shells with and without a correspondingly detached molecular shell may also be explained as follows. For example, there are differences in the shell sizes and masses and in their locations in the Galaxy, when we compare the $\mathrm{CO}$-absent and $\mathrm{CO}$-present sources. The $\mathrm{CO}$-present sources are on average smaller in size, closer to the galactic plane, and located in regions with higher cirrus confusions (see Schöier et al. 2005). Also the current massloss rates are on average smaller for the CO-present sources. While the CO-present sources seem to exhibit mass loss below $10^{-7} M_{\odot} \mathrm{yr}^{-1}$, the CO-absent sources do above it. It is considered that mass-loss rate will once decline to a small value after a thermal pulse and then resume gradually to the rate at the quiescent hydrogen shell-burning phase. The higher current mass-loss rates in the three $\mathrm{CO}$-absent sources may imply a longer periods of time since they experienced the latest thermal pulse, which is comaptible with their larger shell sizes. As to their locations in the Galaxy, higher cirrus confusion would mean denser interstellar medium, which can result in a larger amount of matter to be swept into the shell due to shell-ISM interaction, adding interstellar molecular gas to the detached shell and helping protect 
the circumstellar molecules from photodissociation by the interstellar radiation field.

One of our important findings is that the dust shell of U Hya is confined in a narrow radial range. We estimate that the shell ejection lasted at most in a period between 8000 and 5000 years ago based on the obtained inner radius $\left(\sim 100^{\prime \prime}\right)$ and twice of the half-mass thickness $\left(<50^{\prime \prime}\right)$ if we assume an expansion velocity of $15 \mathrm{~km} \mathrm{~s}^{-1}$ in the shell. This velocity is much higher than the wind velocity of the present-day mass loss of $7 \mathrm{~km} \mathrm{~s}^{-1}$. However, observations of detached CO gas shells show that their outflow velocities are generally higher than the present-day wind speeds (Schöier et al. 2005 and references therein). According to Schöier et al., the gas outflow velocity of a detached CO shell in a carbon star would be $\sim 15 \mathrm{~km} \mathrm{~s}^{-1}$ when the shell size is $0.1 \mathrm{pc}$. Furthermore, it is found that their corresponding detached dust shells are nearly co-located, if not at the same places, in all of the three stars examined so far (U Ant(inner), R Scl, and $\mathrm{U}$ Cam), which indicates the dust is almost comoving with the gas (Maercker et al. 2010; Olofsson et al. 2010). This near colocation is also consistent with the conclusion by Young et al. (1993b). We may be able to expect that the expansion velocity of the dust shell of $\mathrm{U}$ Hya would be at least larger than $\sim 7 \mathrm{~km} \mathrm{~s}^{-1}$, and probably as large as $\sim 15 \mathrm{~km} \mathrm{~s}^{-1}$.

The luminosity of U Hya at the quiescnent H-shell burning phase should be between $\sim(3-6) \times 10^{3} L_{\odot}$. The interpulse period for this luminosity is found to be $(2-1) \times 10^{5}$ years, using the core mass-luminosity relation for low-mass stars $(0.5<$ $M_{\mathrm{c}}<0.66$, where $M_{\mathrm{c}}$ is the core mass in units of solar mass, Boothroyd \& Sackmann 1988a) and core mass-interpulse period relation (Boothryod \& Sackmann 1988b) for the solar abundance. The interpulse period is an order of magnitude longer than the formation age of the detached dust shell of U Hya, which is compatible with the formation scenario discussed here.

SS00 predict that the total mass of the detached shell is $0.007-0.01 M_{\odot}$ when the shell size is $(1-5) \times 10^{17} \mathrm{~cm}$ in a model adjusted for the case of TT Cyg (Olofsson et al. 2000). Schöier et al. (2005) find that the mass-loss eruptions occurred at rates two orders of magnitude higher than those of the preceding phases $\left(10^{-5} M_{\odot} \mathrm{yr}^{-1}\right.$ and $10^{-7} M_{\odot} \mathrm{yr}^{-1}$, respectively) and their duration were as short as a few hundread years in those thin detached $\mathrm{CO}$ shell sources. As given above we deduce that the dust shell in U Hya was formed in a period of at most $(3000)\left(v_{\mathrm{e}} / 15\right)^{-1}$ years, during which dust of $1.1 \times$ $10^{-4}\left(\kappa_{100} / 25\right)^{-1} M_{\odot}\left(\right.$ or total mass of $\left.\sim 10^{-2} M_{\odot}\right)$ was ejected (see Sect. 3). We then find a mean total mass-loss rate in the shell on the order of $\left(10^{-6}-10^{-5}\right) M_{\odot} \mathrm{yr}^{-1}$ (also see Tables 3 and 4). These numbers are compatible with SSOO and Schöier et al.

The two-wind interaction due to a fast wind caused by a thermal pulse seems plausible, given the likely occurrence of a thermal pulse in the recent past in U Hya and rough agreement in the shell mass between U Hya and other sources with extended, detached gas and dust shells.

\subsubsection{Termination shock scenario}

One drawback of the thermal pulse scenario is that $\mathrm{Y} \mathrm{CVn}$ does not show either Tc or other s-process enhancements, while no detailed abundance analysis has been done yet on $\mathrm{U}$ Ant. Although it does not necessarily mean that Y CVn has not experienced a thermal pulse at all, it might suggest that detached dust shells can also form by another mechanism different from the combination of thermal pulse and two-wind interaction. The dust shell of U Hya could be formed by a mechanism that does not require a thermal pulse.
Rowan-Robinson et al. (1986) suggested that excess $100 \mu \mathrm{m}$ emission detected in a few late-type stars by IRAS arose in a shell of dust at the zone where the wind from the star run into the interstellar gas. Young et al. (1993b) also showed that a pileup of the past constant mass-loss flow retained by the ambient interstellar matter could explain the extended dust shell of an evolved star. We note, however, that their results show that the ISM would hardly affect the mass-loss wind at the size of the dust shell of U Hya, $\leq \sim 0.1 \mathrm{pc}$.

Libert et al. (2007) have developed a termination shock model that was compatible with Young et al. (1993a) and only required a moderate constant mass loss to account for the extended, quasi-static H I gas envelope of the carbon star Y CVn. In their model, the inner and outer radii of the circumstellar shell correspond to the locations of termination shock and bow shock, respectively. These very extended envelopes of $\mathrm{H}$ I gas were predicted by Villaver et al. (2002), who explored the dynamical interaction between the stellar winds and the ISM on parsec scales. They found that transient shells were formed with an average lifetime of $\sim 20000$ years by the wind variation associated with the thermal pulses. Their results indicate that the density or velocity structure of the envelopes at great distances $(\geq \sim 0.5 \mathrm{pc})$ is no longer a time series record of mass loss. At the same time, however, their results also show that the matter distribution reflects the mass-loss history at the radius of $\leq \sim 0.1 \mathrm{pc}$, which applies to the case of U Hya.

Gérard \& Le Bertre (2006) have detected H I emission toward 26 evolved stars with both oxygen-rich and carbon-rich chemistries, which includes U Hya. The H I emission from $\mathrm{U}$ Hya extends $32^{\prime}$ in diameter, which is about 8 times more than the dust shell investigated in this paper. This indicates that the $\mathrm{H}$ I gas is not associated directly with the extended dust shell seen in the FIR. However, the envelope size, hence the corrected mass, may be overestimated by the possible down-stream wake to be discussed in Sect. 4.2. It could be the case that they might have detected a tail of the blown-off material from the circumstellar envelope of U Hya by the ISM wind, extending westward, like what has been seen in $o$ Cet (Matthews et al. 2008; Ueta 2008). Detecting such a wake can result in an overestimate of the envelope size. That U Hya is the most extreme case in Fig. 24 of Gérard \& Le Bertre may lend support to such an overestimate.

In testing the termination shock scenario, we consider the ejected mass budget for U Hya. Let us assume a constant mass loss. From the center to the outer edge, the circumstellar shell can be divided into three regions: unperturbed current massloss wind, compressed mass-loss wind, and compressed exterior matter that could have either an interstellar origin or previous episode(s) of mass-loss origin. The latter would be either an RGB mass loss or an accumulation of AGB mass loss during interpulse periods two or more cycles before the current period. There are a termination shock between the former two regions, and a contact discontinuity between the last two. The third region is outlined by a bow shock. Then the total mass in the shell $M_{\text {shell }}$ may be written by $M_{\text {shell }}=\dot{M}\left(t_{\text {form }}-R_{\text {in }} / v_{\mathrm{e}}\right)+(4 / 3) \pi R_{\text {out }}^{3} \rho_{\text {out }}$, where $\dot{M}, t_{\text {form }}, R_{\text {in }}, v_{\mathrm{e}}, R_{\text {out }}$, and $\rho_{\text {out }}$ are the current mass-loss rate, formation age, inner boundary radius, outflow velocity, outer edge radius, and density exterior to the shell, respectively. Here we assume the current mass-loss wind started at the origin of $t_{\text {form. }}$.

On the basis of a formulation given by Ivezic \& Elitzur (1995 and references therein), a steadily blowing wind will pile the ambient gas up into a shell after $t_{\text {form }} \simeq 3.2 \times 10^{9}\left(\dot{M} / n_{\text {out }} / v_{\mathrm{e}}^{3}\right)^{1 / 2} \mathrm{yrs}$, where $\dot{M}$ and $v_{\mathrm{e}}$ are in units of $M_{\odot} \mathrm{yr}^{-1}$ and $\mathrm{km} \mathrm{s}^{-1}$, respectively, 
and $n_{\text {out }}$ is the density of exterior medium by hydrogen atoms in $\mathrm{cm}^{-3}$. Also the location of the contact discontinuity is written as $r_{\text {shell }} \simeq 10^{22}\left(\dot{M} / n_{\text {out }} / v_{\mathrm{e}}\right)^{1 / 2} \mathrm{~cm}$, here. These formulations give numbers compatible with those suggested by Young et al. (1993b). Following Young et al. (1993b) we find an ISM density of $0.7 \mathrm{H} \mathrm{cm}^{-3}$ from the scale height of $100 \mathrm{pc}$ and an ISM density of $2 \mathrm{H} \mathrm{cm}^{-3}$ in the Galactic plane for the galactic latitude of $38^{\circ}$ and a distance of $162 \mathrm{pc}$. Substituting the ISM density for $n_{\text {out }}$ gives $t_{\text {form }}=7.3 \times 10^{4}$ years and $r_{\text {shell }}=1.6 \times 10^{18} \mathrm{~cm}$ that corresponds to $617^{\prime \prime}$ on the sky for the current mass-loss rate of $1.2 \times 10^{-7} M_{\odot} \mathrm{yr}^{-1}$ and the wind velocity of $6.9 \mathrm{~km} \mathrm{~s}^{-1}$ (Olofsson et al. 1993). The obtained $t_{\text {form }}$ and $r_{\text {shell }}$ seem much longer and larger than those for the observed dust shell of U Hya, while the $r_{\text {shell }}$ is comparable to the size of the H I envelope derived by Gérard \& Le Bertre (2006), though the H I size is subject to an overestimate as discussed before. Besides, $t_{\text {form }}$ amounts to a considerable fraction of the interpulse period obtained in the preceding section.

We obtain $M_{\text {shell }}=2.1 \times 10^{-2}\left(=7.4 \times 10^{-3}+1.4 \times 10^{-2}\right) M_{\odot}$ by adopting $R_{\text {out }}=r_{\text {shell }}=1.6 \times 10^{18} \mathrm{~cm}, R_{\text {in }}=2.4 \times 10^{17} \mathrm{~cm}\left(100^{\prime \prime}\right)$, $\rho_{\text {out }}=1.6 \times 10^{-24} \mathrm{~g} \mathrm{~cm}^{-3}$ for the ISM density of $0.7 \mathrm{H} \mathrm{cm}^{-3}$, and $t_{\text {form }}=7.3 \times 10^{4}$ years. This agrees fairly well with our estimate of the total mass in the detached shell of U Hya, though the size difference between the observed dust and the inferred gas is not negligible, whereas Gérard \& Le Bertre (2006) give the $\mathrm{H}$ I envelope mass of $5.5 \times 10^{-3} M_{\odot}$ for the estimated envelope size of $32^{\prime}$, which results in an average mass-loss rate of $0.2 \times$ $10^{-7} M_{\odot} \mathrm{yr}^{-1}$ for $\mathrm{U}$ Hya. The rate seems significantly lower than the current mass-loss rate of $1.2 \times 10^{-7} M_{\odot} \mathrm{yr}^{-1}$ derived from $\mathrm{CO}$ rotational lines.

The size descrepancy may be relaxed by introducing a higher $\rho_{\text {out }}$ (and $n_{\text {out }}$ ) that will allow adjustment of both $t_{\text {form }}$ and $r_{\text {shell }}$ to the observed dust shell of U Hya. Here we adopt $150^{\prime \prime}=3.6 \times 10^{17} \mathrm{~cm}$ for $r_{\text {shell }}$ from Fig. 7. Then we obtain $n_{\text {out }}=13$, which is 19 times higher than the estimated ISM density, and $t_{\text {form }}=1.7 \times 10^{4}$ years. These numbers give $M_{\text {shell }}=$ $3.7 \times 10^{-3}\left(=7.2 \times 10^{-4}+3.0 \times 10^{-3}\right) M_{\odot}$ for $R_{\text {out }}=r_{\text {shell }}$, which seems acceptable. The high $n$ (thus $\rho_{\text {out }}$ ) could be interpreted as the presence of circumstellar matter due to an older mass-loss episode(s) (Libert et al. 2007).

We summarize that the surrounding medium exterior to the constant mass-loss wind must be more than an order of magnitude denser than the local ISM to be compatible with the termination shock scenario.

\subsection{Offset of the shell center}

The shell center is found to be displaced from the central pointlike source by $9^{\prime \prime}$ to the right and $3^{\prime \prime}$ to the bottom on the WIDE$S$ image (Fig. 2) as determined in Sect. 3.1. This gives the radial displacement and position angle (PA) of the shell center with respect to the star of $9^{\prime \prime} .5$ and $-85^{\circ} \pm 15^{\circ}$, respectively, in the equatorial coordinates, whereas the PA of the proper motion of U Hya is $+132^{\circ}$. If $\mathrm{U}$ Hya is moving through an interstellar medium stationary to the sun, the offset should be opposite to the proper motion, PA at $-48^{\circ}$. An additional PA offset of $-37^{\circ}$ may suggest a flow of interstellar matter in the plane of the sky with respect to the Sun (see Ueta et al. 2008, 2010). If so, a downstream wake should appear, not around PA $=-48^{\circ}$, but around $\mathrm{PA}=-85^{\circ} \pm 15^{\circ}$. Indeed we see a sign of excess emission extending west to the edge of the map, $4^{\prime} .5$ from the star, around $\mathrm{PA}=-70^{\circ}$ in the model-subtracted WIDE-S image as shown in Fig. 8. The excess emission is also discernible in the $70 \mu \mathrm{m}$ image of U Hya taken with the Multiband Imaging Photometer

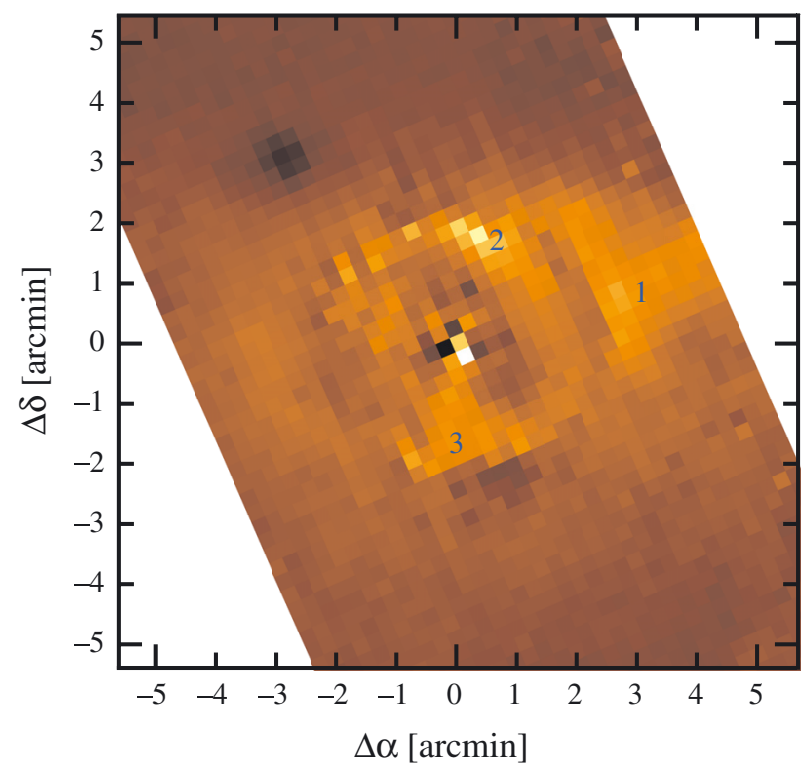

Fig. 8. Model-subtracted WIDE-S image of U Hya (see text) shown in the equatorial coordinates. North is up and east to the left. The axes give the offsets with respect the star. Pixel size is $15^{\prime \prime}$. The subtracted model is the one with $\alpha=-4.5$ in Table 4, arbitrarily multiplied by 0.85 so that the extended components become the most evident. Excess emission (" 1 ") is seen to the east of the shell along PA $\sim-70^{\circ}$ with respect to the star in the equatorial coordinates. Its peak brightness is $\sim 4 \mathrm{MJy} \mathrm{sr}^{-1}$ above the background level. Other excesses at PA $\sim-7^{\circ}$ (" 2 ") and $\sim 180^{\circ}$ ("3") from circular symmetry in the shell brightness distribution is also discernible (see text).

aboard the Spitzer Space Telescope (Geise et al. 2010). Thus, the emission could come from the circumstellar matter around U Hya blown-off by and mixed with the ISM wind.

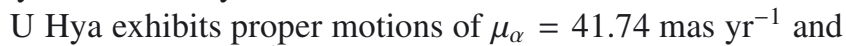

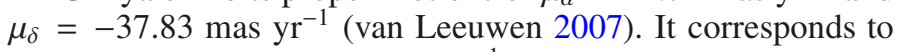
a transverse velocity of $43 \mathrm{~km} \mathrm{~s}^{-1}$ in the plane of the sky for the distance of $162 \mathrm{pc}$. The ISM wind velocity in the plane of the sky projected onto the direction at $\mathrm{PA}=-160^{\circ}$ would be $16 \mathrm{~km} \mathrm{~s}^{-1}$, while the one in the offset direction (here we use $\mathrm{PA}=-70^{\circ}$ ) is unknown. Let us assume the latter is negligible. Then a point stationary to the ISM will be separated from the

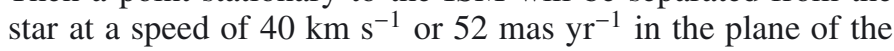
sky in the offset direction. This speed gives an extent of $7^{\prime}$ for the estimated shell age of 8000 years. The extent roughly agrees with the observed extension of the wake of 4'.5. The agreement could be better, since the unknown velocity component along the offset direction would be non-negligible and could be negative (to PA $=+110^{\circ}$ ), which reduces the effective speed of the ISM wind with respect to the star. The line of sight velocity of $\mathrm{U}$ Hya with respect to the local standard of rest, $V_{\mathrm{LSR}}$ is $-30.7 \mathrm{~km} \mathrm{~s}^{-1}$ (Olofsson et al. 1993). The star's motion with respect to the ISM must be stronger than its transverse velocity alone. Further observations with higher sensitivity and spatial resolution in the FIR will reveal further details of the interaction between the mass-loss flow from U Hya and the interstellar matter.

We note that extended dust envelopes with a significant offset have been revealed in M-type Mira variables that are likely oxygen-rich AGB stars: R Hya (Hashimoto et al. 1998; Ueta et al. 2006), $o$ Cet(Mira) by (Ueta 2008) and R Cas (Ueta et al. 2010). They all have been interpreted as bow shock-induced dust emission based on the large offsets of the dust shell centers with 
respect to the stellar positions and their bow-like shapes, which are not the case for U Hya.

We also remark that there are additional emission components extending to the north and south of the star inside the shell in Fig. 8. These extensions, in particular the northern one, probably come from optical depth enhancements in those lines of sight, since the northern brightness enhancement at the rim at the $\mathrm{PA} \sim-7^{\circ}$ in the WIDE-S image (bottom row of Figs. 2 and 8) can also be identified in the $V$-band optical image (Izumiura et al. 2007), in which the overall shape of the shell is quite consistent with that in the WIDE-S image. Since the optical dust shell is presumably seen in scattered star light by dust grains in the shell, at least the northern enhancement is probably caused by an enhanced dust column density in that line of sight. The FIR excess may be a signature of the earliest departure from spherical symmetry in the AGB mass loss. It is worth investigating further.

\section{Conclusions}

We obtained far-infrared images at $65,90,140$, and $160 \mu \mathrm{m}$ of the carbon star U Hya using the Far-Infrared Surveyor onboard AKARI infrared astronomical satellite with a $68.5 \mathrm{~cm}$ aperture diameter. We summarize our findings as follows:

1. A detached spherical dust shell almost centered on the carbon star U Hya was revealed in the four far-infrared bands for the first time by direct imaging. The central star and the shell were decomposed, giving reliable estimates of their farinfrared flux densities for the first time.

2. We used a simple model of a detached dust shell to fit the observed shell images of U Hya. We find that the inner radius of the shell $R_{\text {in }}$ is $101-107^{\prime \prime},(2.5-2.6) \times 10^{17} \mathrm{~cm}$, or $0.08 \mathrm{pc}$ for the distance of $162 \mathrm{pc}$. The half-mass width $\Delta R_{\mathrm{hm}}$ is $16-23^{\prime \prime}$ or $(3.8-5.6) \times 10^{16} \mathrm{~cm}$, which is smaller than the AKARI/FIS spatial resolutions. The power-law in$\operatorname{dex} \beta$ of the dust opacity law $\kappa(\lambda) \propto \lambda^{-\beta}$ as a function of wavelength $\lambda$ is found to be 1.10-1.15. A high value of $6.0-7.0$ is obtained for the power-law index $\alpha$ of the dust density distribution $\rho(r) \propto r^{-\alpha}$ for the radius $r$, though it is not constrained uniquely. It ranges from -4.5 to 7.0. The dust mass in the shell is well constrained to be $(0.9-1.4) \times 10^{-4}\left(\kappa_{100} / 25\right)^{-1} M_{\odot}$, where $\kappa_{100}$ is the dust absorptivity at $100 \mu \mathrm{m}$ in units of $\mathrm{cm}^{2} \mathrm{~g}^{-1}$. This gives a total mass of $\sim 10^{-2}\left(\kappa_{100} / 25\right)^{-1}\left(f_{\mathrm{gd}} / 100\right) M_{\odot}$, where $f_{\mathrm{gd}}$ is the gas-to-dust mass ratio. Dust mass-loss rate at the inner boundary is ill constrained to be (1.8$9.6) \times 10^{-8}\left(\kappa_{100} / 25\right)^{-1}\left(v_{\mathrm{e}} / 15\right) M_{\odot} \mathrm{yr}^{-1}$ corresponding to the unconstrained various $\alpha$, where $v_{\mathrm{e}}$ is the dust outflow velocity in units of $\mathrm{km} \mathrm{s}^{-1}$. This gives a total mass-loss rate of $(1.8-9.6) \times 10^{-6}\left(v_{\mathrm{e}} / 15\right)\left(\kappa_{100} / 25\right)^{-1}\left(f_{\mathrm{gd}} / 100\right) M_{\odot} \mathrm{yr}^{-1}$ at $R_{\text {in }}$, which is more than an order of magnitude higher than the present-day mass-loss rate. A new robust number is also found for the power-law dust density distributions: $\rho\left(118^{\prime \prime}\right)=(1.8-2.4) \times 10^{-24}\left(\kappa_{100} / 25\right)^{-1} \mathrm{~g} \mathrm{~cm}^{-3}$.

3 . We examined whether two existing scenarios for the origin of the detached circumstellar shells are also compatible with the detached dust shell of U Hya: a thermal pulse induced mass-loss eruption with a high velocity followed by two-wind interaction (SS00; Mattsson et al. 2007), and a termination shock caused by the interaction between a constant mass-loss wind and the interstellar matter (Libert et al. 2007). The former scenario is preferred, given the likely occurrence of a thermal pulse in the recent past and a rough agreement in the shell mass between U Hya and other carbon stars with extended, detached gas shells. However, the latter scenario is also viable if the density of ambient matter is an order of magnitude higher than the expected ISM density.

4. The shell center is probably displaced by $\sim 9.5$ with respect to the star. The position angle of the displacement is $-85^{\circ} \pm 15^{\circ}$, which does not align with the direction of the proper motion of $U$ Hya. The offset may be the result of the effects from both the proper motion and the flow of interstellar matter with respect to the sun. The presence of the ISM wind is reinforced by an emission extending along PA $\sim-70^{\circ}$ out to a distance of at least $5^{\prime}$ from the star that could be a wake by the ISM wind.

5. We find excess emission to the north and south of the star inside the shell, which is probably due to optical depth enhancements in those lines of sight. It may be the earliest departure from spherical symmetry in the AGB mass loss.

Acknowledgements. The authors are grateful to the anonymous referee for the careful reading of the manuscript. The referee's valuable comments have improved the manuscript significantly. This research is based on observations with AKARI, a JAXA project with the participation of ESA. This research has made use of the SIMBAD database, operated at the CDS, Strasbourg, France. VizieR (Ochsenbein et al. 2000) is used.

\section{References}

Abia, C., Domínguez, I., Gallino, R., et al. 2002, ApJ, 579, 817

Bedijn, P. J. 1987, A\&A, 186, 136

Boothroyd, A. I., \& Sackmann, I. J. 1988a, ApJ, 328, 641

Boothroyd, A. I., \& Sackmann, I. J. 1988b, ApJ, 328, 653

Bowen, G. H. 1988, ApJ, 329, 299

Draine, B. T. 1981, ApJ, 245, 880

Fleischer, A. J., Gauger, A., \& Sedlmayr, E. 1992, A\&A, 266, 321

Gail, H. P., \& Sedlmayr, E. 1987, A\&A, 171, 197

Geise, K. M., Ueta, T., Speck, A. K., Izumiura, H., \& Stencel, R. E. 2010, BAAS, 42, 364

Gérard, E., \& Le Bertre, T. 2006, AJ, 132, 2566

Groenewegen, M. A. T. 1997, A\&A, 317, 503

Groenewegen, M. A. T., de Jong, T., van der Bliek, N. S., Slijkhuis, S., \& Willems, F. J. 1992, A\&A, 253, 150

Groenewegen, M. A. T., van der Veen, W. E. C. J., \& Matthews, H. E. 1998, A\&A, 338, 491

Hacking, P., Beichman, C., Chester, T., Neugebauer, G., \& Emerson, J. 1985, PASP, 97, 616

Hashimoto, O., Izumiura, H., Kester, D. J. M., \& Bontekoe, T. R. 1998, A\&A, 329,213

Hildebrand, R. H. 1983, Quart. J. R. A. S., 24, 267

Höfner, S., \& Dorfi, E. A. 1997, A\&A, 319, 648

Ivezić, Z., \& Elitzur, M. 1995, ApJ, 445, 415

Izumiura, H., Hashimoto, O., Kawara, K., Yamamura, I., \& Waters, L. B. F. M. 1996, A\&A, 315, L221

Izumiura, H., Waters, L. B. F. M., de Jong, T., et al. 1997, A\&A, 323, 449

Izumiura, H., Nakada, Y., Hashimoto, O., Hayashi, T., \& Mito, H. 2007, in Why Galaxies Care About AGB Stars, ed. F. Kerschbaum, C. Charbonnel, \& R. F. Wing (San Francisco: ASP), 305

Joint IRAS Science Working Group 1988, IRAS catalogs and atlases, The Small Scale Structure Catalog (SSSC) (Washington: US Government Printing Office)

Jura, M. 1986, ApJ, 303, 327

Kawada, M., Baba, H., Barthel, P. D., et al. 2007, PASJ, 59, S389

Kerschbaum, F., Olofsson, H., \& Hron, J. 1996, A\&A, 311, 273

Kerschbaum, F., Ladjal, D., Ottensamer, R., et al. 2010, A\&A, 518, L140

Knapp, G. R., \& Morris, M. 1985, ApJ, 292, 640

Kwok, S., Purton, C. R., \& Fitzgerald, P. M. 1978, ApJ, 219, L125

Le Bertre, T., \& Gérard, E. 2004, A\&A, 419, 549

Libert, Y., Gérard, E., \& Le Bertre, T. 2007, MNRAS, 380, 1161

Matthews, L. D., Libert, Y., Gérard, E., Le Bertre, T., \& Reid, M. J. 2008, ApJ, 684,603

Maercker, M., Olofsson, H., Eriksson, K., Gustafsson, B., Schöier, F. L. 2010, A\&A, 511, A37

Mauron, N. \& Huggins, P. J. 2000, A\&A, 359, 707

Mattsson, L., Höfner, S., \& Herwig, F. 2007, A\&A, 470, 339 
Meixner, M., Campbell, M. T., Welch, W. J., \& Likkel, L. 1998, ApJ, 509, 392 Murakami, H., Baba, H., Barthel, P., et al. 2007, PASJ, 59, S369

Nakada, Y. 1988, PASJ, 40, 331

Ochsenbein, F., Bauer, P., \& Marcout, J. 2000, A\&AS, 143, 221

Olofsson, H., Eriksson, K., \& Gustafsson, B. 1988, A\&A, 196, L1

Olofsson, H., Carlström, U., Eriksson, K., Gustafsson, B., \& Wilson, L. A. 1990, A\&A, 230, L13

Olofsson, H., Eriksson, K., Gustafsson, B., \& Carlström, U. 1993, ApJS, 87, 267 Olofsson, H., Bregman, P., Eriksson, K., \& Gustafsson, B. 1996, A\&A, 311, 587

Olofsson, H., Bergman, P., Lucas, R., Eriksson, K., Gustafsson, B., \& Bieging, J. H. 2000, A\&A, 353, 583

Olofsson, H., Maercker, M., Eriksson, K., Gustafsson, B., \& Schöier, F. L. 2010, A\&A, 515, A27

Peery, B. F., Jr. 1971, ApJ, 163, L1

Pilbratt, G. L., Riedinger, J. R., Passvogel, T., et al. 2010, A\&A, 518, L1

Rowan-Robinson, M., Lock, T. D., Walker, D. W., \& Harris, S. 1986, MNRAS, 222,273

Samus, N. N., Durlevich, O. V., et al. 2010, General Catalogue of Variable Stars, Institute of Astronomy of Russian Academy of Sciences and Sternberg, State Astronomical Institute of the Moscow State University

Schöier, F. L., Lindqvist, M., \& Olofsson, H. 2005, A\&A, 436, 633

Shirahata, M., Matsuura, S., Hasegawa, S., et al. 2009, PASJ, 61, 737

Sopka, R. J., Hildebrand, R., Jaffe, D. T., et al. 1985, ApJ, 294, 242

Steffen, M., \& Schönberner, D. 2000, A\&A, 357, 180 (SS00)
Steffen, M., Szczerba, R., \& Schoenberner, D. 1998, A\&A, 337, 149

Tsuji, T. 1981, J. Astrophys. Astron., 2, 95

Ueta, T. 2008, ApJ, 687, L33

Ueta, T., Speck, A. K., Stencel, R. E., et al. 2006, ApJ, 648, L39

Ueta, T., Izumiura, H., Yamamura, I., et al. 2008, PASJ, 60, S407

Ueta, T., Stencel, R. E., Yamamura, I., et al. 2010, A\&A, 514, A16

Utsumi, K. 1985, in Cool Stars with Excesses of Heavy Elements, ed. M. Jascheck, \& P. C. Keenan (Dordrecht: Reidel) 243 van Leeuwen, F. 2007, A\&A, 474, 653

van der Veen, W. E. C. J., \& Habing, H. J. 1988, A\&A, 194, 125 Vassiliadis, E., \& Wood, P. R. 1993, ApJ, 413, 641

Villaver, E., García-Segura, G., \& Manchado, A. 2002, ApJ, 571, 880

Wareing, C. J. 2007, MNRAS, 382, 1233

Waters, L. B. F. M., Loup, C., Kester, D. J. M., Bontekoe, Tj. R., \& de Jong, T. 1994, A\&A, 281, L1

Werner, M. W., Roellig, T. L., Low, F. J., et al. 2004, ApJS, 154, 1

Willems, F. J., \& de Jong, T. 1988, A\&A, 196, 173

Willson, L. A. 2000, ARA\&A, 38, 573

Woitke, P. 2006, A\&A, 460, L9

Yamamura, I., Makiuti, S., Ikeda, N., et al. 2010, AKARI-FIS Bright Source Catalogue Release note Version 1.0

Young, K., Phillips, T. G., \& Knapp, G. R. 1993a, ApJS, 86, 517

Young, K., Phillips, T. G., \& Knapp, G. R. 1993b, ApJ, 409, 725

Zijlstra, A. A., \& Weinberger, R. 2002, ApJ, 572, 1006 